\title{
Irreducible background of gravitational waves from a cosmic defect network: Update and comparison of numerical techniques
}

\author{
Daniel G. Figueroa $\odot,{ }^{1, *}$ Mark Hindmarsh $\odot,{ }^{2,3, \dagger}$ Joanes Lizarraga $\odot^{4,+}$ and Jon Urrestilla $\odot^{4, \S}$ \\ ${ }^{1}$ Instituto de Física Corpuscular (IFIC), University of Valencia-CSIC, E-46980 Valencia, Spain \\ ${ }^{2}$ Physics Department, University of Helsinki and Helsinki Institute of Physics, \\ P. O. Box 64, FI-00014 Helsinki, Finland \\ ${ }^{3}$ Department of Physics \& Astronomy, University of Sussex, Brighton BN1 9QH, United Kingdom \\ ${ }^{4}$ Department of Theoretical Physics, University of the Basque Country UPV-EHU, 48040 Bilbao, Spain
}

(Received 15 July 2020; accepted 9 October 2020; published 13 November 2020)

\begin{abstract}
Cosmological phase transitions in the early Universe may produce relics in the form of a network of cosmic defects. Independently of the order of a phase transition, topology of the defects, and their global or gauge nature, the defects are expected to emit gravitational waves (GWs) as the network energymomentum tensor adapts itself to maintaining scaling. We show that the evolution of any defect network (and for that matter any scaling source) emits a GW background with spectrum $\Omega_{\mathrm{GW}} \propto f^{3}$ for $f \ll f_{0}$, $\Omega_{\mathrm{GW}} \propto 1 / f^{2}$ for $f_{0} \lesssim f \lesssim f_{\text {eq }}$, and $\Omega_{\mathrm{GW}} \propto$ const (i.e., exactly scale invariant) for $f \gg f_{\text {eq }}$, where $f_{0}$ and $f_{\text {eq }}$ denote respectively the frequencies corresponding to the present and matter-radiation equality horizons. This background represents an irreducible emission of GWs from any scaling network of cosmic defects, with its amplitude characterized only by the symmetry-breaking scale and the nature of the defects. Using classical lattice simulations we calculate the GW signal emitted by defects created after the breaking of a global symmetry $O(N) \rightarrow O(N-1)$. We obtain the GW spectrum for $N$ between 2 and 20 with two different techniques: integrating over unequal-time correlators of the energy-momentum tensor, updating our previous work on smaller lattices, and for the first time, comparing the result with the real-time evolution of the tensor perturbations sourced by the same defects. Our results validate the equivalence of the two techniques. Using cosmic microwave background upper bounds on the defects' energy scale, we discuss the difficulty of detecting this GW background in the case of global defects.
\end{abstract}

DOI: 10.1103/PhysRevD.102.103516

\section{INTRODUCTION}

The direct detection [1-5] of gravitational waves (GWs) by Advanced LIGO [6] and Advanced VIRGO [7], represents a milestone in astronomy, and has opened a new window for exploring the Universe. Other GW detectors have just started observation (KAGRA [8]), or have been approved (LIGOIndia $[9,10])$. A next generation of detectors is already being planned, including the Einstein Telescope [11] on the ground, and the Laser Interferometer Space Antenna (LISA) [12] and Taiji $[13,14]$ in space. Other proposals for space-based detectors, include the Deci-hertz Interferometer Gravitational wave Observatory (DECIGO) $[15,16]$, and Big Bang Observatory (BBO) [17]. Sufficiently energetic processes in the early Universe leave behind characteristic signatures in the form of stochastic GW backgrounds, which are beginning to be constrained from the ground [18,19]. Space-based

\footnotetext{
*daniel.figueroa@ific.uv.es †mark.hindmarsh@helsinki.fi

*joanes.lizarraga@ehu.eus

jon.urrestilla@ehu.eus
}

detectors will place more stringent constraints on early Universe scenarios, and have greater potential to detect cosmological sources [20,21]. Gravitational waves are, in fact, the most promising cosmic relic to probe many of the currently unknown details of the early Universe. It is important therefore to characterize all possible stochastic backgrounds in order to achieve a better understanding of a future detection.

Whenever there is an energy-momentum tensor with a nonzero transverse-traceless (TT) part, GWs are expected to be emitted. In the absence of any source, GWs are also generated quantum mechanically during inflation, with an almost scale-invariant spectrum [22-25]. Depending on the underlying high-energy physics modeling of inflation, active sources may also be present, generating GWs with a large amplitude and blue tilt [26-34]. After inflation, a kination-dominated phase may also induce a large blue tilt in the inflationary GW background [35-40], whereas nonequilibrium phenomena can lead to a strong production of GWs, from particle production at (p)reheating [41-54] and oscillon dynamics [55-60], to strong first-order phase transitions [61-72] and cosmic string networks [73-80]. 
For a review on early Universe GW cosmological backgrounds, see Ref. [20].

In this paper we study the GW background sourced by a self-similar energy-momentum tensor. Self-similarity, or scaling, means that the length scale of the energymomentum distribution is proportional to the cosmic time. Scaling is exhibited by cosmic defects [81,82], which are a natural by-product of a phase transition in the early Universe. Cosmic defects also create anisotropies in the cosmic microwave background (CMB) [83-87]. The search for cosmic defects in the $\mathrm{CMB}$ corresponds precisely to studying the imprint of the metric perturbations created by the defects. The B-mode polarization signal in the CMB is partly created by the tensor metric perturbation that will form the GW background we study in this work.

Cosmic defects also exhibit other potentially observable effects: non-Gaussianity [88-90] and spectral distortions [91] in the CMB, lensing events [92,93], and cosmic rays from the decay of strings into particle radiation [94-103]. In the case of Nambu-Goto strings, a stochastic background of GWs is expected from the oscillations of the resulting loops, chopped off from the main string network through cosmic history [73,78-80,104-111]. The search for this background places the most stringent bounds on the energy scale of Nambu-Goto strings; see e.g., Refs. [18,77,111-113].

As mentioned, cosmic defects are formed in early Universe phase transitions, which are usually described as a spontaneous symmetry-breaking process, driven by some scalar field(s) acquiring a nonzero expectation value within a vacuum manifold $\mathcal{M}$. If the manifold is topologically nontrivial, i.e., has a nontrivial homotopy group $\pi_{n}(\mathcal{M}) \neq \mathcal{I}$, topologically nontrivial field configurations will arise, producing strings for $n=1$, monopoles for $n=2$, and textures for $n=3$ [114]. For higher $n$, the symmetry-breaking field is not topologically obstructed from reaching the vacuum manifold at any point in spacetime, and nontopological field configurations arise. In cases when the symmetry broken is global, all nonconstant field configurations produce energy-momentum, and are loosely referred to as global defects. When the broken symmetry is gauged and the nontrivial homotopy groups have $n=0,1$ or 2 , local defects appear. Cosmic strings, whether global or gauged, as well as any type of global defect exhibit scaling behavior, sufficiently long after the completion of the phase transition that created them $[81,82,115,116]$. All cases, topological or not, local or global, will be referred to as cosmic defects.

In a previous paper [117], which will be referred to as Paper I from now on, we clarified the origin of the scale invariance of the GW background emitted by the selfordering process of nontopological textures, arising after a global phase transition [118-121]. We further generalized the result, showing that any scaling source at the era of radiation domination (RD) produces a $\mathrm{GW}$ background with a scale-invariant energy density power spectrum.
In the case of cosmic defects, we emphasized that this is not related to their particular topology, or to the order of the phase transition, or to the global or local nature of the symmetry-breaking process that generated them. It is just a consequence of scaling and being in RD. Using lattice simulations as an input, in Paper I we also numerically calculated the GW amplitude from a system of global $O(N)$ defects, providing evidence that the numerical result converges to the analytical result calculated in the large- $N$ limit in Refs. [120,122].

While in Paper I we clarified the origin of the scale invariance of the GW spectrum and determined how the GW signal approaches (as we increase $N$ ) the analytical large- $N$ approximate result, various pertinent questions remain to be answered: how does the GW spectrum change when the GWs are emitted during matter domination (MD)? Can the GW background leave an observable imprint in the cosmic microwave background? Can it be detected with pulsar timing arrays (PTAs) and with directdetection GW interferometers? Given the potential relevance of a detection of this background, it seems particularly pertinent to improve the details on the prediction of the signal itself. Another important question, which we intend to address elsewhere with these methods, is the GW spectrum when the defects arise from the breaking of a gauge symmetry, as in the case of local strings.

In the present paper, we update and complement the results from Paper I. First, we extend our prediction to a broader range of frequencies, studying the GWs emitted by a scaling source during both RD and MD. This introduces a new feature into the spectrum, which does not remain scale invariant within the entire frequency range. We also study the GW spectrum at superhorizon scales. In particular, we find that the energy density power spectrum scales as $h^{2} \Omega_{\mathrm{GW}} \propto f^{3}$ for $f \ll f_{0}$, where $f_{0}$ is the frequency today corresponding to the present horizon. The spectrum reaches a maximum at $f=f_{0}$, and between $f=f_{0}$ and $f=f_{\text {eq }}$ scales as $h^{2} \Omega_{\mathrm{GW}} \propto 1 / f^{2}$, where $f_{\text {eq }}$ is the frequency today corresponding to the horizon at the moment of matterradiation equality. Eventually, for $f \gg f_{\text {eq }}$, the spectrum settles down to a scale-invariant amplitude $h^{2} \Omega_{\mathrm{GW}} \propto$ const, as reported in Paper I.

Second, we update the numerical input used in Paper I based on the extraction of the unequal-time correlators (UETCs) of the transverse-traceless part of the energymomentum tensor from field theory lattice simulations; namely we use new simulations with a larger volume, from which we obtain UETCs with a wider spectral range. We also present the reconstruction of the GW spectrum based on the sum over a weighted eigenvalue-eigenvector decomposition coming from the diagonalization of the UETC.

Third, and most importantly, we present a complementary numerical calculation of the gravitational-wave energy density power spectrum, which allows two nontrivial checks of our results: 
(1) We obtain the GW spectrum by following the realtime evolution of the tensor metric perturbations, as they are continuously sourced by the defect network itself. We compare for the first time the GW spectra from the UETC method with those produced by realtime evolution from the same energy-momentum source. We discuss the circumstances under which a good agreement is found within an appropriate spectral range. The success of this comparison provides a validation of both methods, suggesting that the use of either method should be equally acceptable in future numerical GW computations.

(2) The prediction of the GW power spectra from defect networks (say scale invariant in RD) is rooted in the assumption of perfect scaling. We have checked that by "switching on" the defect source term in the equations of motion of the tensor perturbations before the defect network has reached the scaling regime, this does not produce the predicted spectrum from scaling as it should. The reason behind this is that in such cases the spectrum reflects the highly random initial field configuration, which eventually prevents the signal from forming the expected scaling profile. This result highlights the importance of initiating the GW evolution only when the network is in scaling.

Finally we discuss the amplitude today of the GW backgrounds from different defect networks, based on our simulations. We compare our results with previous studies of GW production from global defects available in the literature.

We note that the case $N=2$, corresponding to global strings, is anomalous, in that our updated GW power is of order a factor $\sim 2$ bigger than the value given in Paper I. We argue that this is connected with the special difficulties in assessing the scaling of the network, as discussed in Ref. [123]. This case is especially relevant as it can be connected e.g., with string-inspired models $[124,125]$ that enjoy (approximate) global symmetries with low $N$, as well as with axion-like dark matter candidates, where a network of global strings is naturally expected to be produced; see e.g., Ref. [126]. Further work is required in order to make a robust prediction of its GW signal.

The paper is organized as follows. In Sec. II we review the basic aspects of stochastic GW backgrounds with sources. In Sec. III we turn our attention to GWs sourced by scaling seeds. We derive previous and new aspects of the frequency dependence of the GW spectrum, depending on the cosmic epoch. For comparison, we review briefly the analytical calculation of the GW spectrum in RD, in the case of a global phase transition $O(N) \rightarrow O(N-1)$ with $N \gg 1$ (further details are shown in Appendix A). In Sec. IV we discuss some aspects of the methodology of our lattice simulations, and we present the definition and extraction of the tensor UETC from them. We then give numerical examples of the GW energy density spectra obtained with this technique, using our new numerical simulations of global defects, based on a $O(N) \rightarrow O(N-1)$ symmetry breaking with arbitrary $N$. We also show the reconstruction of the GW spectrum through partial summation of weighted terms obtained from the diagonalization of the tensor UETC. In Sec. V we present our numerical results for the GW energy density spectrum from the same simulations introduced in Sec. IV, but obtained from the real-time evolution (in real space) of the tensor perturbations, while being sourced by the defect network. We discuss the limitations to reconstruct the spectrum by this method, and the circumstances required to reach a good agreement with the method based on UETCs. In Sec. VI we summarize our results, highlight some of the technical difficulties involved in our numerical calculations, and discuss the difficulty to detect this GW background.

We work in $\hbar=c=1$ units, where $M_{\mathrm{Pl}}=1 / \sqrt{G} \approx$ $1.22 \times 10^{19} \mathrm{GeV}$ is the Planck mass, and $G$ is Newton's constant. Summation over repeated indices is assumed.

\section{GRAVITATIONAL WAVES}

We will study the evolution of the fields and the gravitational waves, when the Universe is well described by a spatially flat Friedman-Lemaitre-Robertson-Walker (FLRW) metric, sourced by a perfect fluid. Including the relevant metric perturbation, the line element is written as

$$
d s^{2}=a^{2}(t)\left[-d t^{2}+\left(\delta_{i j}+h_{i j}\right) d x^{i} d x^{j}\right],
$$

where $a(t)$ is the scale factor, $t$ is conformal time, and the metric perturbations $h_{i j}$ are transverse $\left(\partial_{i} h_{i j}=0\right)$ and traceless $\left(h_{i}^{i}=0\right)$.

Splitting the Einstein equations into background and linearized equations, the GW equations of motion (EOMs) in a FLRW background are (see e.g., Ref. [20])

$$
\ddot{\bar{h}}_{i j}(\mathbf{x}, t)-\left(\nabla^{2}+\frac{\ddot{a}(t)}{a(t)}\right) \bar{h}_{i j}(\mathbf{x}, t)=16 \pi G a(t) \Pi_{i j}^{\mathrm{TT}}(\mathbf{x}, t),
$$

where we have introduced a conformal redefinition of the tensor perturbations $\bar{h}_{i j}(\mathbf{x}, t)=a(t) h_{i j}(\mathbf{x}, t)$, and dots denote derivatives with respect to the conformal time. The source $\Pi_{i j}^{\mathrm{TT}}$ is the TT part of the anisotropic stress tensor $\Pi_{i j}$, which we define below. The conditions $\partial_{i} \Pi_{i j}^{\mathrm{TT}}=$ $\Pi_{i i}^{\mathrm{TT}}=0$ hold for $\forall \mathbf{x}, \forall t$. Either in RD or in MD, or in general for a scale factor with a power-law behavior in time, it holds that $\ddot{a} / a \sim \mathcal{H}^{2}$, where $\mathcal{H} \equiv \dot{a} / a$ is the (comoving) Hubble rate. Hence, the term $\ddot{a} / a$ is negligible at subhorizon scales $k \gg \mathcal{H}$, and therefore we will drop it from now on. The EOM of subhorizon modes in Fourier space can then be written as 


$$
\ddot{\bar{h}}_{i j}(\mathbf{k}, t)+k^{2} \bar{h}_{i j}(\mathbf{k}, t)=16 \pi G a(t) \Pi_{i j}^{\mathrm{TT}}(\mathbf{k}, t),
$$

where $\mathbf{k}$ is the comoving wave number and $k=|\mathbf{k}|$ is its modulus. The solution to Eq. (3) is given by a convolution with the Green's function associated to a free wave operator in Minkowski spacetime, $G_{>}\left(k, t-t^{\prime}\right)=k^{-1} \sin \left[k\left(t-t^{\prime}\right)\right]$. That is, at times $t>t_{I}$, where $t_{I}$ is an initial time with no gravitational waves, $h_{i j}\left(\mathbf{k}, t_{I}\right)=\dot{h}_{i j}\left(\mathbf{k}, t_{I}\right)=0$, we obtain

$$
\begin{aligned}
h_{i j}(k, t) & =\frac{\bar{h}_{i j}(k, t)}{a(t)} \\
& =\frac{16 \pi G}{k a(t)} \int_{t_{I}}^{t} d t^{\prime} a\left(t^{\prime}\right) \sin \left[k\left(t-t^{\prime}\right)\right] \Pi_{i j}^{\mathrm{TT}}\left(k, t^{\prime}\right) .
\end{aligned}
$$

Obtaining the TT part of a tensor in configuration space amounts to a nonlocal operation. It is more convenient to do it in Fourier space, where a projector filtering out only the TT degrees of freedom of a tensor can be easily written down. The GW source can then be written as

$$
\Pi_{i j}^{\mathrm{TT}}(\mathbf{k}, t)=\Lambda_{i j, l m}(\hat{\mathbf{k}}) \Pi_{l m}(\mathbf{k}, t),
$$

where $\Lambda_{i j, l m}(\hat{\mathbf{k}})$ is a projection operator defined as

$$
\begin{aligned}
\Lambda_{i j, l m}(\hat{\mathbf{k}}) & \equiv P_{i l}(\hat{\mathbf{k}}) P_{j m}(\hat{\mathbf{k}})-\frac{1}{2} P_{i j}(\hat{\mathbf{k}}) P_{l m}(\hat{\mathbf{k}}), \\
P_{i j} & =\delta_{i j}-\hat{k}_{i} \hat{k}_{j}, \quad \hat{k}_{i}=k_{i} / k .
\end{aligned}
$$

Thanks to the fact that $P_{i j} \hat{k}_{j}=0$ and $P_{i j} P_{j m}=P_{i m}$, one can easily see that the transverse-traceless conditions in Fourier space, $k_{i} \Pi_{i j}^{\mathrm{TT}}(\hat{\mathbf{k}}, t)=\Pi_{i i}^{\mathrm{TT}}(\hat{\mathbf{k}}, t)=0$, are satisfied at any time.

The anisotropic stress tensor $\Pi_{\mu \nu}$ describes the deviation of an energy-momentum tensor $T_{\mu \nu}$ with respect to a perfect fluid. The spatial-spatial components read

$$
\Pi_{i j} \equiv T_{i j}-p g_{i j}
$$

where $p$ is the homogeneous background pressure and $g_{i j}=a^{2}(t)\left(\delta_{i j}+h_{i j}\right)$ is the spatial-spatial FLRW perturbed metric. In the scenarios we consider in this paper the energy density is dominated by a homogeneous and isotropic perfect fluid. The energy-momentum of this background has spatial-spatial components $T_{i j}^{\mathrm{pf}}=p g_{i j}$, where the pressure is either one third of the energy density (RD) or zero (MD).

On top of this there is a subdominant contribution from cosmic defects, which have their own energy-momentum tensor $T_{i j}^{\text {def }}$. Hence, in these scenarios, the (spatialspatial components of the) total energy-momentum tensor are given by $T_{i j}=T_{i j}^{\mathrm{pf}}+T_{i j}^{\mathrm{def}}$. It is clear then that
$\Pi_{i j}=T_{i j}^{\mathrm{def}}$, so that the active source of GWs in our case is the TT part of the cosmic defects' energy-momentum tensor.

\section{A. Spectrum of gravitational waves}

Expanding the Einstein equations to second order in the tensor perturbations, one recognizes that the energy density of a $\mathrm{GW}$ background is given by [127]

$$
\begin{aligned}
\rho_{\mathrm{GW}}(t)= & \frac{1}{32 \pi G a^{2}(t)}\left\langle\dot{h}_{i j}(\mathbf{x}, t) \dot{h}_{i j}(\mathbf{x}, t)\right\rangle_{V} \\
\equiv & \frac{1}{32 \pi G a^{2}(t)} \frac{1}{V} \int_{V} d \mathbf{x} \dot{h}_{i j}(\mathbf{x}, t) \dot{h}_{i j}(\mathbf{x}, t) \\
= & \frac{1}{32 \pi G a^{2}(t)} \int \frac{d \mathbf{k}}{(2 \pi)^{3}} \frac{d \mathbf{k}^{\prime}}{(2 \pi)^{3}} \dot{h}_{i j}(\mathbf{k}, t) \dot{h}_{i j}^{*}\left(\mathbf{k}^{\prime}, t\right) \\
& \times \frac{1}{V} \int_{V} d \mathbf{x} e^{-i \mathbf{x}\left(\mathbf{k}-\mathbf{k}^{\prime}\right)},
\end{aligned}
$$

where $\langle\ldots\rangle_{V}$ is a spatial average over a sufficiently large comoving volume $V$ encompassing all the relevant wavelengths of the $h_{i j}$ perturbations. In the limit $k V^{1 / 3} \gg 1$, $\int_{\mathrm{V}} d \mathbf{x} e^{-i \mathbf{x}\left(\mathbf{k}-\mathbf{k}^{\prime}\right)} \rightarrow(2 \pi)^{3} \delta^{(3)}\left(\mathbf{k}-\mathbf{k}^{\prime}\right)$, and hence

$\rho_{\mathrm{GW}}(t)=\frac{1}{32 \pi G a^{2}(t) V} \int \frac{d \mathbf{k}}{(2 \pi)^{3}} \dot{h}_{i j}(\mathbf{k}, t) \dot{h}_{i j}^{*}(\mathbf{k}, t)$.

The GW energy density spectrum per logarithmic interval is defined as

$$
\rho_{\mathrm{GW}}(t) \equiv \int \frac{d \rho_{\mathrm{GW}}}{d \log k} d \log k,
$$

$\frac{d \rho_{\mathrm{GW}}}{d \log k}=\frac{k^{3}}{(4 \pi)^{3} G a^{2}(t) V} \int \frac{d \Omega_{k}}{4 \pi} \dot{h}_{i j}(\mathbf{k}, t) \dot{h}_{i j}^{*}(\mathbf{k}, t)$,

where $d \Omega_{k}$ represents a solid angle element in $\mathbf{k}$ space.

In our case, GWs are created from a network of cosmic defects. As the symmetry-breaking process that originates the defects is a random process, we cannot predict the exact location of each cosmic defect. However, we can still describe the stochastic distribution that characterizes the defect network. The spatial distribution of the GW will therefore be assumed to also be stochastic, following the random distribution of the defects. Applying the ergodic hypothesis, we can replace $\langle\ldots\rangle_{V}$ by an ensemble average $\langle\ldots\rangle$ over realizations. The stochastic background of GWs can then be described by 


$$
\begin{aligned}
\rho_{\mathrm{GW}}= & \frac{1}{32 \pi G a^{2}(t)}\left\langle\dot{h}_{i j}(\mathbf{x}, t) \dot{h}_{i j}(\mathbf{x}, t)\right\rangle \\
= & \frac{1}{32 \pi G a^{2}(t)} \int \frac{d \mathbf{k}}{(2 \pi)^{3}} \frac{d \mathbf{k}^{\prime}}{(2 \pi)^{3}} e^{i \mathbf{x}\left(\mathbf{k}-\mathbf{k}^{\prime}\right)} \\
& \times\left\langle\dot{h}_{i j}(\mathbf{k}, t) \dot{h}_{i j}^{*}\left(\mathbf{k}^{\prime}, t\right)\right\rangle .
\end{aligned}
$$

The expectation value in the second line of Eq. (13), assuming statistical homogeneity and isotropy, can be written as

$$
\left\langle\dot{h}_{i j}(\mathbf{k}, t) \dot{h}_{i j}^{*}\left(\mathbf{k}^{\prime}, t\right)\right\rangle \equiv(2 \pi)^{3} P_{\dot{h}}(k, t) \delta^{(3)}\left(\mathbf{k}-\mathbf{k}^{\prime}\right),
$$

so that we can write

$$
\rho_{\mathrm{GW}}(t)=\frac{1}{(4 \pi)^{3} G a^{2}(t)} \int \frac{d k}{k} k^{3} P_{\dot{h}}(k, t) .
$$

From here we define the $G W$ energy density power spectrum as

$$
\frac{d \rho_{\mathrm{GW}}}{d \log k}(k, t)=\frac{1}{(4 \pi)^{3} G a^{2}(t)} k^{3} P_{\dot{h}}(k, t),
$$

which will be referred to as the GW power spectrum (or simply as the GW spectrum).

We can obtain $P_{\dot{h}}(k, t)$ with the help of Eq. (4), by first writing

$\dot{h}_{i j}(\mathbf{k}, t)=\frac{16 \pi G}{k a(t)} \int_{t_{I}}^{t} d t^{\prime} a\left(t^{\prime}\right) \mathcal{G}\left(k\left(t-t^{\prime}\right)\right) \Pi_{i j}^{\mathrm{TT}}\left(\mathbf{k}, t^{\prime}\right)$,

with $\quad \mathcal{G}\left(k\left(t-t^{\prime}\right)\right) \equiv\left(k \cos \left[k\left(t-t^{\prime}\right)\right]-\mathcal{H} \sin \left[k\left(t-t^{\prime}\right)\right]\right)$. This leads to

$$
\begin{aligned}
P_{\dot{h}}(k, t)= & \frac{(16 \pi G)^{2}}{k^{2} a^{2}(t)} \int_{t_{I}}^{t} d t^{\prime} \int_{t_{I}}^{t} d t^{\prime \prime} a\left(t^{\prime}\right) a\left(t^{\prime \prime}\right) \\
& \times \mathcal{G}\left(k\left(t-t^{\prime}\right)\right) \mathcal{G}\left(k\left(t-t^{\prime \prime}\right)\right) \Pi^{2}\left(k, t^{\prime}, t^{\prime \prime}\right),
\end{aligned}
$$

where we have introduced the UETC of the TT part of the anisotropic stress $\Pi_{i j}^{\mathrm{TT}}$,

$$
\left\langle\Pi_{i j}^{\mathrm{TT}}(\mathbf{k}, t) \Pi_{i j}^{\mathrm{TT}}\left(\mathbf{k}^{\prime}, t^{\prime}\right)\right\rangle \equiv(2 \pi)^{3} \Pi^{2}\left(k, t, t^{\prime}\right) \delta^{(3)}\left(\mathbf{k}-\mathbf{k}^{\prime}\right) .
$$

Once GW production ends, GWs propagate as free waves, each mode oscillating with period $T_{k}=2 \pi / k$. We therefore need to take a time average over the product of $\mathcal{G}\left(\mathbf{k}, t, t^{\prime}\right)$ functions,

$$
\begin{aligned}
\left\langle\mathcal{G}\left(\mathbf{k}, t, t^{\prime}\right) \mathcal{G}\left(\mathbf{k}, t, t^{\prime \prime}\right)\right\rangle_{T_{k}} & \equiv \frac{1}{T_{k}} \int_{t}^{t+T_{k}} d \tilde{t} \mathcal{G}\left(\mathbf{k}, \tilde{t}, t^{\prime}\right) \mathcal{G}\left(\mathbf{k}, \tilde{t}, t^{\prime \prime}\right) \\
& =\frac{1}{2}\left(k^{2}+\mathcal{H}^{2}(t)\right) \cos \left[k\left(t^{\prime}-t^{\prime \prime}\right)\right]
\end{aligned}
$$

Replacing $\mathcal{G}\left(\mathbf{k}, t, t^{\prime}\right) \mathcal{G}\left(\mathbf{k}, t, t^{\prime \prime}\right)$ by $\left\langle\mathcal{G}\left(\mathbf{k}, t, t^{\prime}\right) \mathcal{G}\left(\mathbf{k}, t, t^{\prime \prime}\right)\right\rangle_{T_{k}}$ in Eq. (18), and taking into account that at subhorizon scales $\left(k^{2}+\mathcal{H}^{2}(t)\right) \approx k^{2}$, we arrive at

$$
\begin{aligned}
P_{\dot{h}}= & \frac{(16 \pi G)^{2}}{2 a^{2}(t)} \int_{t_{I}}^{t} d t^{\prime} \int_{t_{I}}^{t} d t^{\prime \prime} a\left(t^{\prime}\right) a\left(t^{\prime \prime}\right) \\
& \times \cos \left[k\left(t^{\prime}-t^{\prime \prime}\right)\right] \Pi^{2}\left(k, t^{\prime}, t^{\prime \prime}\right) .
\end{aligned}
$$

Plugging Eq. (21) into Eq. (16), we finally find the GW energy density power spectrum of a stochastic background of GWs (at subhorizon scales) as

$$
\begin{aligned}
\frac{d \rho_{\mathrm{GW}}}{d \log k}(k, t)= & \frac{2}{\pi} \frac{G k^{3}}{a^{4}(t)} \int_{t_{I}}^{t} d t^{\prime} \int_{t_{I}}^{t} d t^{\prime \prime} a\left(t^{\prime}\right) a\left(t^{\prime \prime}\right) \\
& \times \cos \left[k\left(t^{\prime}-t^{\prime \prime}\right)\right] \Pi^{2}\left(k, t^{\prime}, t^{\prime \prime}\right) .
\end{aligned}
$$

For convenience, we can also normalize the GW energy density spectrum to the critical density $\rho_{c} \equiv 3 H^{2} / 8 \pi G$, obtaining

$$
\begin{aligned}
\Omega_{\mathrm{GW}}(k, t) \equiv & \frac{1}{\rho_{c}} \frac{d \rho_{\mathrm{GW}}}{d \log k} \\
= & \frac{16 G^{2} k^{3}}{3 H^{2} a^{4}(t)} \int_{t_{I}}^{t} d t^{\prime} \int_{t_{I}}^{t} d t^{\prime \prime} a\left(t^{\prime}\right) a\left(t^{\prime \prime}\right) \\
& \times \cos \left[k\left(t^{\prime}-t^{\prime \prime}\right)\right] \Pi^{2}\left(k, t^{\prime}, t^{\prime \prime}\right)
\end{aligned}
$$

\section{GRAVITATIONAL WAVES FROM SCALING SEEDS}

Based on causality and dimensional grounds, Ref. [118] originally argued that the field dynamics following after a global phase transition should generate an approximately scale-invariant background of GWs. The amplitude of such a background was estimated with the GW quadrupole approximation, without any reference to the number of components $N$ of the corresponding symmetry-breaking field.

In the context of a phase transition driven by the breaking of a global $O(N)$ symmetry into a $O(N-1)$ group, even though the field equations are nonlinear, analytic calculations can be carried out in the $N \gg 1$ limit, describing the evolution of the nontopological global defects that emerge after the phase transition; see Ref. [115]. Within such a context and using a full treatment of the tensor metric perturbation (i.e., without resorting to the quadrupole approximation), Refs. $[119,120]$ demonstrated that in the 
large- $N$ limit, an exact scale-invariant background of GWs is generated (during $\mathrm{RD}$ ) by the self-ordering dynamics of the nontopological global defects arising after the $O(N) \rightarrow$ $O(N-1)$ symmetry breaking. On the numerical side, Ref. [121] studied lattice simulations after a second-order phase transition, concluding that the global defects created in that case generate a GW background consistent with scale invariance, even though the numerical spectra exhibited some tilt and oscillatory fluctuations.

In this section we will generalize the above results, and derive and discuss the common aspects of the spectral shape of the stochastic GW background emitted by any network of cosmic defects in a scaling regime (which will be simply referred to as scaling seeds). In particular, in Sec. III A, we first review our findings from Paper I during $\mathrm{RD}$, and then we extend the results to the production of GWs from the evolution of scaling seeds during MD. In Sec. III B we characterize the GW background at superhorizon scales for both RD and MD. In Sec. III C we discuss the overall spectral shape of the GW background spanned over all frequencies, and in particular the form of the resulting redshifted spectrum today. Finally, in Sec. III D, we review the analytic estimation of the GW signal emitted by self-ordering scalar fields based on the large- $N$ limit of global defects. We postpone the presentation of our numerical results from lattice simulations to Secs. IV and V, where two different numerical methods for obtaining the spectrum of GWs emitted by a network of cosmic defects will be presented. There we will also compare the analytic formulation of this section with the outcome from the numerical simulations.

\section{A. GW spectrum at subhorizon scales}

The origin of the scale invariance of the GW background emitted by the self-ordering process of nontopological defects was clarified in Paper I. There it was demonstrated that any scaling source with a nonvanishing transversetraceless energy-momentum tensor always produces a background of GWs during RD, with an exact scaleinvariant energy density power spectrum. As emphasized in Paper I, the result is just a consequence of the defects' scaling behavior and of being in RD.

Let us recall that once we know the UETC of the tensor anisotropic stress $\Pi^{2}\left(k, t_{1}, t_{2}\right)$ [Eq. (19)], we can compute the spectrum of GWs emitted by simply plugging $\Pi^{2}\left(k, t_{1}, t_{2}\right)$ into Eq. (22). In the case of a defect network, the correlator $\Pi^{2}\left(k, t_{1}, t_{2}\right)$ can be obtained, from field theory simulations $[87,128-132]$. In the specific case of nontopological defects arising after spontaneous symmetry breaking of a global $O(N), \Pi^{2}\left(k, t_{1}, t_{2}\right)$ can be also estimated analytically in the large- $N$ limit, as we will review in Sec. III D.

Before we consider the explicit form of the UETC from a defect network, let us recall the most fundamental property of any network of cosmic defects: whenever cosmic defects are created during a phase transition, the resulting defect network (after the phase transition is completed) gradually enters into a scaling regime, where the number density of defects per comoving Hubble volume $\sim 1 / \mathcal{H}^{3} \sim t^{3}$, remains invariant through cosmic history $[81,82,114]$. Once in the scaling regime, the UETC can only depend on $k$ through the dimensionless variables $x_{1}=k t_{1}$ and $x_{2}=k t_{2}$. From dimensional analysis it is forced to take the form

$$
\Pi^{2}\left(k, t_{1}, t_{2}\right)=\frac{4 v^{4}}{\sqrt{t_{1} t_{2}}} \mathcal{U}\left(k t_{1}, k t_{2}\right),
$$

where $v$ is the vacuum expectation value (VEV) in the broken state of the scalar fields (the factor 4 is a convention to match the tensor UETC of Ref. [133]).

Using the scaling form of the correlator,

$$
\begin{aligned}
\Omega_{\mathrm{GW}}(k, t)= & \frac{64 k^{2}}{3 H^{2} a^{4}(t)}\left(\frac{v}{M_{\mathrm{Pl}}}\right)^{4} \\
& \times \int d x_{1} d x_{2} \frac{a_{1} a_{2}}{\sqrt{x_{1} x_{2}}} \cos \left(x_{1}-x_{2}\right) \mathcal{U}\left(x_{1}, x_{2}\right),
\end{aligned}
$$

where $a_{1} \equiv a\left(x_{1} / k\right), a_{2} \equiv a\left(x_{2} / k\right)$.

Before the late-time accelerated expansion of the Universe, but after the era of electron-positron annihilation, the scale factor can be written as

$$
\begin{aligned}
a(t) & =a_{\mathrm{eq}}\left(\left[(\sqrt{2}-1)\left(t / t_{\mathrm{eq}}\right)+1\right]^{2}-1\right) \\
& =a_{0}^{3} \Omega_{\mathrm{mat}}^{(0)} \frac{H_{0}^{2} t^{2}}{4}+a_{0}^{2} \sqrt{\Omega_{\mathrm{rad}}^{(0)}} H_{0} t,
\end{aligned}
$$

where $a_{\mathrm{eq}}$ is the scale factor at the time of matter-radiation equality, $t_{\mathrm{eq}}$. In the second expression we have used the integral representation

$$
\begin{aligned}
a_{0} H_{0} t_{\mathrm{eq}} & =\int_{z_{\mathrm{eq}}}^{\infty} \frac{d z}{\sqrt{\Omega_{\mathrm{rad}}^{(0)}(1+z)^{4}+\Omega_{\mathrm{mat}}^{(0)}(1+z)^{3}}} \\
& =\frac{2(\sqrt{2}-1)}{\left(1+z_{\mathrm{eq}}\right)} \frac{1}{\sqrt{\Omega_{\mathrm{rad}}^{(0)}}} .
\end{aligned}
$$

Changes in the number of relativistic degrees of freedom (d.o.f.) during $\mathrm{RD}$ can be easily taken into account by correcting the solution deep in the radiation era as

$$
a_{\mathrm{RD}}(t)=a_{0}^{2} \sqrt{\Omega_{\mathrm{rad}}^{(0)}} H_{0} \int \mathcal{R}_{t^{\prime}} d t^{\prime},
$$

where 


$$
\mathcal{R}_{t} \equiv\left(\frac{g_{s, 0}}{g_{s, t}}\right)^{4 / 3}\left(\frac{g_{\mathrm{th}, t}}{g_{\mathrm{th}, 0}}\right)
$$

where $g_{s, t}$ and $g_{\mathrm{th}, t}$ are the entropic and thermal energy density numbers of relativistic d.o.f. at time $t$. For most of cosmic history, $g_{s, t} \simeq g_{\mathrm{th}, t}$, and it is a good approximation to treat $\mathcal{R}$ as a piecewise constant function. We take $\mathcal{R}_{t} \simeq$ $\mathcal{R}_{\mathrm{QCD}} \simeq 0.39$ before the quark-gluon $\mathrm{QCD}$ phase transition $t<t_{\mathrm{QCD}}, \mathcal{R}_{t} \simeq \mathcal{R}_{\mathrm{e}^{-} \mathrm{e}^{+}} \simeq 0.81$ between $\mathrm{QCD}$ and electronposition annihilation $t_{\mathrm{QCD}}<t<t_{\mathrm{e}^{-} \mathrm{e}^{+}}$, and $\mathcal{R}_{t} \simeq \mathcal{R}_{0}=1$ after electron-position annihilation $t>t_{\mathrm{e}^{-}} \mathrm{e}^{+}$. The scale factor Eq. (26) during RD can then be approximated as

$$
a_{\mathrm{RD}}(t) \simeq \sqrt{\Omega_{\mathrm{rad}}^{(0)}} a_{0}^{2} H_{0} \mathcal{R}_{*} t,
$$

with $\mathcal{R}_{*} \simeq \mathcal{R}_{\mathrm{QCD}}, \mathcal{R}_{\mathrm{e}^{-} \mathrm{e}^{+}}$or $\mathcal{R}_{0}$, depending on the time $t$.

Plugging this behavior into Eq. (25), leads to a subhorizon spectrum of $\mathrm{GW}$, for modes that become subhorizon $x \equiv k t \gg 1$ during $\mathrm{RD}$, as

$$
\begin{aligned}
\Omega_{\mathrm{GW}}(x, t) & =\Omega_{\mathrm{rad}}(t)\left(\frac{v}{M_{\mathrm{Pl}}}\right)^{4} \frac{\mathcal{R}_{*}}{\mathcal{R}_{t}} F_{\mathrm{RD}}^{[\mathcal{U}]}(x), \\
F_{\mathrm{RD}}^{[\mathcal{U}]}(x) & \equiv \frac{64}{3} \int^{x} d x_{1} \int^{x} d x_{2} \sqrt{x_{1} x_{2}} \cos \left(x_{1}-x_{2}\right) \mathcal{U}\left(x_{1}, x_{2}\right),
\end{aligned}
$$

where $\Omega_{\mathrm{rad}}(t)=1$ while $t \ll t_{\mathrm{eq}}$, and $\Omega_{\mathrm{rad}}(t)<1$ for $t>t_{\text {eq }}$. At subhorizon scales, $\mathcal{U}\left(x_{1}, x_{2}\right)$ is peaked near $x_{1}=x_{2} \equiv x$, and decays along the diagonal as a power law $\propto x^{-p}$, where $p$ is a positive real number; see e.g., Ref. [116]. Hence the convergence of the integration is guaranteed as long as the decay is fast enough, i.e., $p>2$. In such a case $F_{\mathrm{RD}}^{[\mathrm{U}]}(x)$ becomes more and more insensitive to its upper bound of integration, asymptotically approaching a constant value for $x \gg 1$. In other words $F_{\mathcal{U}}(x \gg 1)$ approaches the constant $F_{\mathrm{RD}}^{(\infty)} \equiv F_{\mathrm{RD}}^{[\mathcal{U}]}(x \rightarrow \infty)$. As a consequence of this, the GW spectrum at subhorizon wavelengths becomes scale invariant.

For every type of defect there is a characteristic function $\mathcal{U}\left(x_{1}, x_{2}\right)$, and thus a well-determined value $F_{\mathrm{RD}}^{(\infty)}$, which gives the magnitude of the $\mathrm{GW}$ spectrum. Its value today, with the traditional reference value for the Hubble rate $H_{0}=100 h_{0} \mathrm{~km} \mathrm{~s}^{-1} \mathrm{Mpc}^{-1}$, is

$$
h_{0}^{2} \Omega_{\mathrm{GW}}^{(0)}(k)=h_{0}^{2} \Omega_{\mathrm{rad}}^{(0)}\left(\frac{v}{M_{\mathrm{Pl}}}\right)^{4} \mathcal{R}_{*} F_{\mathrm{RD}}^{(\infty)} .
$$

The GW background produced during RD by the evolution of any network of defects in the scaling regime is therefore exactly scale invariant, modulo a mild stepwise change in $\mathcal{R}_{*}$ due to the evolution of the number of relativistic species in thermal equilibrium. The amplitude of the GW background today is suppressed by the fraction of relativistic species $\Omega_{\mathrm{rad}}^{(0)}$, and is proportional to the fourth power the VEV as $\left(v / M_{\mathrm{Pl}}\right)^{4}$. It also depends on the shape of the UETC (and hence on the type of defect), which ultimately modulates the amplitude through $F_{\mathrm{RD}}^{(\infty)}$.

Equation (31) summarizes the theoretical results from Paper I about GW emission from RD, incorporating now as a new element the change in the number of relativistic species. Next we generalize the analysis to the emission of GWs during MD. For times when the radiation component is completely subdominant, $t \gg t_{\text {eq }}$, the scale factor Eq. (26) can be written approximately as $a(t) \simeq \frac{1}{4} a_{0}^{3} \Omega_{\mathrm{mat}}^{(0)} H_{0}^{2} t^{2}$. As soon as the UETC during MD is scaling, it can be written again as in Eq. (24). For simplicity we assume scaling is maintained for $t \geq t_{\text {eq. }}$ Using Eq. (25), together with Eq. (27), the spectrum of $\mathrm{GW}$ at subhorizon scales $x \equiv k t \gg 1$ during MD reads

$$
\Omega_{\mathrm{GW}}(x, t)=\Omega_{\mathrm{rad}}(t)\left(\frac{v}{M_{\mathrm{Pl}}}\right)^{4} \frac{k_{\mathrm{eq}}^{2}}{k^{2}} F_{\mathrm{MD}}^{[\mathcal{U}]}(x),
$$

where $x_{\text {eq }} \equiv k t_{\text {eq }}, k_{\text {eq }} \equiv 1 / 2 t_{\text {eq }}$, and

$$
\begin{aligned}
F_{\mathrm{MD}}^{[\mathcal{U}]}(x) \equiv & \frac{64}{3}(\sqrt{2}-1)^{2} \\
& \times \int_{x_{\mathrm{eq}}}^{x} d x_{1} \int_{x_{\mathrm{eq}}}^{x} d x_{2}\left(x_{1} x_{2}\right)^{3 / 2} \cos \left(x_{1}-x_{2}\right) \mathcal{U}\left(x_{1}, x_{2}\right) .
\end{aligned}
$$

By construction $k_{\text {eq }}$ corresponds to the mode with half wavelength $\pi / k_{\text {eq }}$ equal to the horizon $1 / t_{\text {eq }}$ at the time of matter-radiation equality. We can redshift this spectrum today as

$$
\begin{aligned}
h_{0}^{2} \Omega_{\mathrm{GW}}^{(0)}(k) & \equiv \frac{1}{\rho_{\mathrm{c}}}\left(\frac{d \rho_{\mathrm{GW}}}{d \log k}\right) \\
& =h_{0}^{2} \Omega_{\mathrm{rad}}^{(0)}\left(\frac{v}{M_{\mathrm{Pl}}}\right)^{4}\left(\frac{k_{\mathrm{eq}}}{k}\right)^{2} F_{\mathrm{MD}}^{(\infty)} .
\end{aligned}
$$

\section{B. GW spectrum at superhorizon scales}

Let us note that Eqs. (31) and (34) correspond only to the GW energy density spectrum for modes well inside the current horizon, during $\mathrm{RD}$ and $\mathrm{MD}$, respectively. The energy density carried by GWs is only meaningful for sub-Hubble modes, because the notion of an energymomentum tensor associated to GWs requires that the wavelengths of the GWs are much smaller than the characteristic length scale of the background metric (see e.g., Ref. [127]). In the case of the FLRW background, a characteristic length scale at any moment is the causal horizon, which is given (modulo factors of order unity) by the instantaneous Hubble radius. The universe is however 
expected to be homogeneous and isotropic beyond the causal horizon, so in principle we can formally extend the notion of GW energy density spectrum at super-Hubble scales $x \equiv k t \ll 1$. The time scale for the oscillation of a given superhorizon mode is however much larger than the age of the universe, $T_{k}=2 \pi / k=(2 \pi / x) t \gg t$, so there is no sense in averaging over oscillations as in Eq. (20). Instead, our starting point must be Eq. (18) with the functions $\mathcal{G}\left(k, t, t^{\prime}\right), \mathcal{G}\left(k, t, t^{\prime \prime}\right)$ evaluated in the superHubble limit $k t, k t^{\prime}, k t^{\prime \prime} \ll 1$.

In order to proceed we note that the Green's function (4) is only valid for sub-Hubble modes $x \gg 1$. Hence, we must derive the general Green's function for the full differential equation (2). For a power-law expansion rate $a(t) \propto t^{p}$, we find

$$
h_{i j}(k, t)=\frac{16 \pi G}{k a(t)} \int_{t_{I}}^{t} d t^{\prime} a\left(t^{\prime}\right) G_{>}^{(p)}\left(x, x^{\prime}\right) \Pi_{i j}^{\mathrm{TT}}\left(k, t^{\prime}\right)
$$

where

$$
G_{>}^{(p)}\left(x, x^{\prime}\right) \equiv x x^{\prime}\left\{j_{p-1}\left(x^{\prime}\right) y_{p-1}(x)-j_{p-1}(x) y_{p-1}\left(x^{\prime}\right)\right\},
$$

and $j_{p}(x), y_{p}(x)$ are spherical Bessel functions of the first and second kind. In terms of the functions $G_{>}^{(p)}$, the Green's functions for $\dot{h}$ are

$$
\mathcal{G}^{(p)}\left(k, t, t^{\prime}\right)=a(t) \frac{d}{d t} \frac{G_{>}^{(p)}\left(x, x^{\prime}\right)}{a(t)} .
$$

For $\mathrm{RD}(p=1)$ we have

$$
G_{>}^{(1)}\left(x, x^{\prime}\right)=\sin \left(x-x^{\prime}\right),
$$

while for $\mathrm{MD}(p=2)$ we obtain

$$
G_{>}^{(2)}\left(x, x^{\prime}\right)=\frac{1}{x x^{\prime}}\left[\left(1+x x^{\prime}\right) \sin \left(x-x^{\prime}\right)-\left(x-x^{\prime}\right) \cos \left(x-x^{\prime}\right)\right] .
$$

The subhorizon Green's function used in Eq. (4) therefore can be applied at arbitrary scales in RD, which follows from $a^{\prime \prime}(t)=0$, but only at large $x$ and $x^{\prime}$ in MD.

We are now ready to obtain $\mathcal{G}\left(k, t, t^{\prime}\right) \equiv$ $a(t) \frac{d}{d t}\left(G_{>}\left(x, x^{\prime}\right) / a(t)\right)$ at superhorizon scales, for an arbitrary power-law expansion rate $a(t) \propto t^{p}$. We find $\mathcal{G}\left(k, t, t^{\prime}\right) \rightarrow k\left(1-\mathcal{H}\left(t-t^{\prime}\right)\right)$, where $\mathcal{H}=p / t$. In particular, for RD and MD we have

$$
\mathcal{G}\left(k, t, t^{\prime}\right) \underset{k t, k t^{\prime} \ll 1}{\longrightarrow} \begin{cases}k\left(t^{\prime} / t\right) & \mathrm{RD}, \\ k\left[-1+2\left(t^{\prime} / t\right)\right] & \mathrm{MD} .\end{cases}
$$

We can now consider Eq. (18) to represent a valid formal expression for the energy density spectrum of GWs at super-Hubble scales, as long as we use the expressions of $\mathcal{G}\left(k, t, t^{\prime}\right)$ given in Eq. (38). This implies that Eq. (22) is also valid at superhorizon scales as long as we replace $\cos \left(k\left(t^{\prime}-t^{\prime \prime}\right)\right)$ by either expression from Eq. (38). The energy density spectrum for scaling seeds (25) is then valid as well at super-Hubble scales, as long as we replace $\cos \left(x_{1}-x_{2}\right)$ by $x_{1} x_{2} / x^{2}$ (for $\mathrm{RD}$ ) or by $(-1+$ $\left.2 x_{1} / x\right)\left(-1+2 x_{2} / x\right)$ (for MD). Alternatively, identical replacements can be done in the specialized expressions for RD [Eq. (30)] and MD [Eq. (32)].

In order to find the scale dependence of the spectrum at super-Hubble scales, the upper bound on the integration (33) is taken $x \ll 1$. At superhorizon scales, the UETC scales as $\mathcal{U}\left(x_{1}, x_{2}\right) \simeq \mathcal{U}_{S H}=$ const for $x_{1} \simeq x_{2}$, and $\mathcal{U}\left(x_{1}, x_{2}\right) \ll \mathcal{U}_{S H}$ otherwise [134]. Then, when we perform the integrals in $F_{\mathrm{RD}}^{[\mathcal{U}]}(x)$ and $F_{\mathrm{MD}}^{[\mathcal{U}]}(x)$, we can take $\mathcal{U}\left(x_{1}, x_{2}\right)$ out of the integrand, and substitute it simply by a constant. We therefore obtain that the GW energy density spectrum at super-hubble scales during $\mathrm{RD}$, scales as

$$
\Omega_{\mathrm{GW}}^{[\mathrm{RD}]}(x \ll 1) \propto\left[\int x \ll 1 d x^{\prime} \sqrt{x^{\prime}}\left(\frac{x^{\prime}}{x}\right)\right]^{2} \propto x^{3},
$$

whereas for $\mathrm{MD}$, it scales like

$$
\begin{aligned}
\Omega_{\mathrm{GW}}^{[\mathrm{MD}]}(x \ll 1) & \propto\left[\frac{k_{\mathrm{eq}}}{k} \int^{x \ll 1} d x^{\prime} x^{\prime 3 / 2}\left(2 \frac{x^{\prime}}{x}-1\right)\right]^{2} \\
& \propto\left(t / t_{\mathrm{eq}}\right)^{2} x^{3} .
\end{aligned}
$$

\section{GW spectrum at all scales (at RD and today)}

According to our previous discussion, the GW energy density spectrum can be formally extended to super-Hubble scales as functions going as $\Omega_{\mathrm{GW}}(x \ll 1) \propto x^{3} \propto(k / \mathcal{H})^{3}$, independently of the expansion rate $a(t) \propto t^{p}$. Hence, at a fixed time during $\mathrm{RD}$, the overall spectrum scales as $\Omega_{\mathrm{GW}}(x \ll 1) \propto k^{3}$ for superhorizon modes, and turns into a scale-invariant spectrum $\Omega_{\mathrm{GW}}(x \gg 1) \propto k^{0}$ for the modes that have already crossed the horizon. Later on, at a given moment during MD, the spectrum scales as $\Omega_{\mathrm{GW}}(x \ll 1) \propto$ $k^{3}$ for superhorizon modes, reaches a maximum at the horizon scale, decreases as $\Omega_{\mathrm{GW}}\left(1 \ll x \ll x_{\mathrm{eq}}\right) \propto k^{-2}$ for scales that crossed during MD, and eventually settles down to a scale-invariant amplitude $\Omega_{\mathrm{GW}}\left(x \gg x_{\mathrm{eq}}\right) \propto k^{0}$ for modes that crossed the horizon during RD.

In Fig. 1 we plot the full spectrum during RD [cf. Eq. (30)]

$$
\Omega_{\mathrm{GW}}^{(\mathrm{RD})}(k, t)=\Omega_{\mathrm{rad}}(t)\left(\frac{v}{M_{\mathrm{Pl}}}\right)^{4} \frac{\mathcal{R}_{*}}{\mathcal{R}_{t}} F_{\mathrm{RD}}^{[\mathcal{U}]}(x),
$$

computed with the aid of UETCs in scaling (for $N=4$ ) that we obtain from lattice simulations in Sec. IV. We scale out $\mathcal{R}_{*} / \mathcal{R}_{t}$ from the plot so that the steps due to the changing of the number of relativistic d.o.f. are not shown, and hence we are left with exact power laws. By evaluating $F_{\mathrm{RD}}^{[\mathcal{U}]}(x)$ at all values $x=k / \mathcal{H}$, we obtain a continuous spectrum 

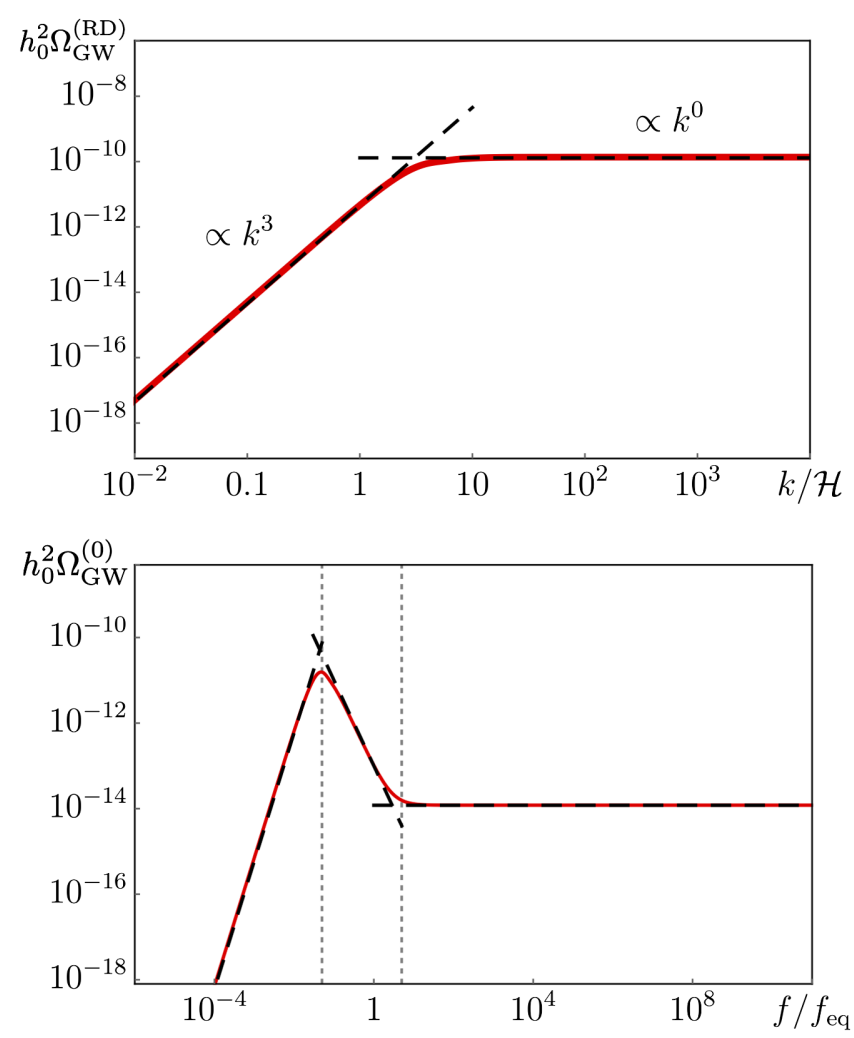

FIG. 1. Top: Instantaneous spectrum of GWs during RD, $h_{0}^{2} \Omega_{\mathrm{GW}}^{(\mathrm{RD})}(k, t)$, plotted as a function of $x=k / \mathcal{H}$, assuming no change in the effective number of relativistic degrees of freedom between production and the time of evaluation $\left(\mathcal{R}_{*} / \mathcal{R}_{t}=1\right)$. Bottom: Redshifted gravitational-wave spectrum today $h_{0}^{2} \Omega_{\mathrm{GW}}^{(0)}$ (using $\mathcal{R}_{*}=1$ ). The dashed vertical lines represent, from left to right, the frequency $f_{0}$ of the present horizon (indicating the maximum of the spectrum), and the transition frequency $f_{*}=$ few $\times f_{\text {eq }}$ that signals the appearance of the high-frequency plateau.

around horizon-crossing scales $x \sim 1$, smoothly interpolating the two asymptotic regimes $F_{\mathrm{RD}}^{[\mathcal{U}]}(x \gg 1) \rightarrow F_{\mathrm{RD}}^{(\infty)}$ at sub-Hubble scales, and $F_{\mathrm{RD}}^{[\mathcal{U}]}(x \ll 1) \propto k^{3}$ at super-Hubble scales. Let us note that even though during $\mathrm{RD}, \Omega_{\mathrm{rad}}(t)=1$ holds by definition, it is nonetheless convenient to maintain such a factor in Eq. (41), as it controls the dilution of the GW spectrum once RD ends [see e.g., Eq. (31), which describes the redshifted plateau amplitude today, suppressed by the current fraction of radiation to total energy density in the Universe $\Omega_{\text {rad }}^{(0)} \ll 1$ ].

Analogously, we plot in Fig. 1 the full redshifted spectrum at the present time (recall we are ignoring the effect of dark energy), by using the superposition of GW spectra obtained separately for RD and MD,

$$
\begin{aligned}
h_{0}^{2} \Omega_{\mathrm{GW}}^{(0)}= & h_{0}^{2} \Omega_{\mathrm{rad}}^{(0)}\left(\frac{v}{M_{\mathrm{Pl}}}\right)^{4} \\
& \times\left(\mathcal{R}_{*} F_{\mathrm{RD}}^{(\infty)} \Theta\left(x-x_{\mathrm{eq}}\right)+\frac{k_{\mathrm{eq}}^{2}}{k^{2}} F_{\mathrm{MD}}^{[\mathcal{U}]}(x)\right),
\end{aligned}
$$

so that by evaluating $F_{\mathrm{MD}}^{[\mathrm{U}]}(x)$ continuously at all values of $x$, we obtain a smooth spectrum around horizon-crossing scales $x \sim 1$, interpolating the super-Hubble regime $F_{\mathrm{RD}}^{[\mathcal{U}]}(x \ll 1) \propto k^{3}$ and the MD crossing scales regime $F_{\mathrm{RD}}^{[\mathcal{U}]}\left(1 \ll x \ll x_{\mathrm{eq}}\right) \propto k^{-2}$, eventually matching smoothly around $x \sim x_{\mathrm{eq}}$ with the $\mathrm{RD}$ crossing scales regime $F_{\mathrm{RD}}^{[\mathcal{U}]}\left(x \gg x_{\mathrm{eq}}\right) \rightarrow F_{\mathrm{RD}}^{(\infty)}$. We choose $\mathcal{R}_{*}=1$ for simplicity in the bottom panel of Fig 1.

Note that in obtaining the full GW spectrum today $h_{0}^{2} \Omega_{\mathrm{GW}}^{(0)}$, we have approximated the transition from RD to $\mathrm{MD}$ at $t_{\mathrm{eq}}$ as instantaneous. The true interpolation of the spectrum between the regime $\Omega_{\mathrm{GW}} \propto k^{-2}$ for modes emitted during $\mathrm{MD}$, and the regime $\Omega_{\mathrm{GW}} \propto$ const for modes emitted during RD, would have small differences from that depicted in the bottom panel of Fig. 1. However, as appreciated in the figure itself, the transition between these two regimes takes place at a frequency slightly larger ${ }^{1}$ than the frequency associated to the horizon scale at the moment of matter-radiation equality. The latter corresponds to a very large scale, and hence to a very small frequency today, which can be obtained as $f_{\text {eq }}=\frac{1}{2 \pi} \frac{k_{\text {eq }}}{a_{0}}=\frac{1}{2} \frac{a_{\mathrm{eq}}}{a_{0}} H_{\mathrm{eq}} \simeq$ $\frac{1}{2}\left(1+z_{\text {eq }}\right)^{1 / 2} H_{0}$, leading to

$$
f_{\text {eq }} \simeq 6.6 \times 10^{-17} \mathrm{~Hz},
$$

where we have used $k_{\text {eq }} \equiv \pi a_{\text {eq }} H_{\text {eq }}, H_{0} / H_{\text {eq }} \simeq\left(a_{\text {eq }} / a_{0}\right)^{3 / 2}$, $a_{0} / a_{\mathrm{eq}} \simeq 1+z_{\mathrm{eq}} \simeq 3400$ and $H_{0} \simeq 70 \mathrm{~km} / \mathrm{s} / \mathrm{Mpc}$. Equation (43) immediately informs us about an important aspect of the GW background we are studying: only the plateau part of the spectrum, emitted during $\mathrm{RD}$, is relevant for direct observation. ${ }^{2}$ This is the case for PTA experiments or current/planned direct-detection interferometer experiments, as the typical frequencies accessible to the former are around $\sim 10^{-8} \mathrm{~Hz}$, whereas the frequency range of the latter spans from $\sim 10^{-4}$ to $\sim 10^{3} \mathrm{~Hz}$ (with huge frequency gaps in between). We further discuss the detectability of this GW background in Sec. VI.

\section{Analytical calculation of the GW background in the large- $N$ limit of a global symmetry breaking}

In a global theory where an $O(N)$ symmetry is spontaneously broken into $O(N-1)$, even though the field equations are nonlinear, analytic calculations are possible

\footnotetext{
${ }^{1}$ In Sec. V we will show that modes evolving during RD only form the plateau once they have became sufficiently small compared to the horizon scale. This explains why the transition scale between the $k^{-2}$ and $k^{0}$ regimes in the spectrum corresponds to a slightly shorter scale than the horizon at matter-radiation equality, i.e., to a frequency $f_{*}=\alpha f_{\text {eq }}$ where $\alpha \gtrsim 1$ is a constant somewhat larger than unity.

${ }^{2}$ This does not apply to the $\mathrm{CMB}$, where the small-frequency part $f \lesssim f_{\text {eq }}$ of the GW spectrum can leave an imprint in the form of temperature and polarization anisotropies.
} 
in the limit of large $N \gg 1[115,135]$. The starting point is an $N$-component scalar field $\Phi=\left(\phi_{1}, \phi_{2}, \ldots, \phi_{N}\right)^{\mathrm{T}} / \sqrt{2}$ with Lagrangian

$$
-\mathcal{L}=\left(\partial_{\mu} \Phi\right)^{\mathrm{T}}\left(\partial^{\mu} \Phi\right)+\lambda\left(|\Phi|^{2}-v^{2} / 2\right)^{2}+\mathcal{L}_{\text {int }},
$$

where $\lambda$ is a dimensionless self-coupling, $v$ is the VEV in the broken phase, $|\Phi|^{2}=\frac{1}{2} \sum_{a} \phi_{a}^{2}$, and $\mathcal{L}_{\text {int }}$ represents some interaction with other degrees of freedom (e.g., a thermal bath or other scalar fields). When due to the dynamics (here unspecified) $\mathcal{L}_{\text {int }}$ cannot compensate the tachyonic mass in the potential any further, the $O(N)$ symmetry is spontaneously broken to $O(N-1)$. As a result, $\Phi$ is driven to the vacuum manifold, given by $|\Phi(\mathbf{x}, t)|^{2}=v^{2} / 2$. Due to causality, in regions separated by a comoving distance larger than the comoving horizon the values of $\Phi(\mathbf{x}, t)$ and $\Phi\left(\mathbf{x}^{\prime}, t\right)$ must be uncorrelated. As a consequence, gradient energy density is generated between disconnected regions. For $N \gg 1$, the dynamics of the Goldstone modes can be well described by a nonlinear sigma model, where we force the vacuum constraint $\sum_{a} \phi_{a}^{2}(\mathbf{x}, t)=v^{2}$ by a Lagrange multiplier. This approximation is very good for physical scales much larger than $m^{-1} \equiv 1 /(\sqrt{\lambda} v)$. At large scales the field components are free to wander around in the vacuum manifold, giving rise to a gradient energy density which will generate GWs on those scales.

Even though the EOMs of the $N$ field components are nonlinear, in practice the self-ordering dynamics of the fields can be described approximately by a linearized EOM when $N \gg 1$ [115]. A simple analytical solution of the field dynamics can then be found, showing explicitly that the self-ordering dynamics of the defects actually exhibit scaling. Once the field solution is known, one can also calculate analytically, again in the large- $N$ limit, the GW power spectrum emitted by the self-ordering dynamics of the nontopological defects. The full details of the calculation of the GW spectrum can be found in Ref. [120], and for completeness we present a schematic derivation in Appendix A. Here we just quote the resulting GW spectral amplitude (today) for modes emitted during RD [120,122]

$$
h_{0}^{2} \Omega_{\mathrm{GW}}^{(0)}(f) \simeq \frac{650}{N} h_{0}^{2} \Omega_{\mathrm{rad}}^{(0)}\left(\frac{v}{M_{\mathrm{Pl}}}\right)^{4},
$$

which, as expected for $\mathrm{RD}$, corresponds to a scale-invariant background (ignoring the change in the number of relativistic d.o.f.), with a spectral amplitude just characterized by a dimensionless number. ${ }^{3}$ There is no dependence either

\footnotetext{
${ }^{3}$ While the original number found in Ref. [120] was 511/N, after improving the numerical integration in Ref. [122], and testing the result against different accuracy levels and schemes of integration, it was found that a more correct number is $650 / N$. The expressions given in either Ref. [120] or Ref. [122] did not consider the change in the number of relativistic d.o.f.
}

on the self-coupling $\lambda$ of the symmetry-breaking field, because the effective theory of the Goldstone modes, responsible for the creation of the GWs is a nonlinear $\sigma$ model, and the coupling disappears when the scalar mode is integrated out. Finally, the fact that the GW signal decreases with $N$ is also expected, as the larger the number of field components, the smaller the gradients between them, and hence the smaller the GWs emitted. We can identify the value of $F_{\mathrm{RD}}^{(\infty)}$ in the large- $N$ analytical calculation (45) as

$$
\left.F_{\mathrm{RD}}^{(\infty)}\right|_{N \rightarrow \infty} \simeq \frac{650}{N}
$$

In Sec. IV we will compare this number and its dependence on $N$ with the actual numerical values of $F_{\mathrm{RD}}^{(\infty)}$ calculated with the input of UETCs obtained from numerical lattice simulations of the dynamics after the spontaneous global breaking of $O(N)$ into $O(N-1)$. As expected, the numerical results approach the analytical amplitude for $N \gg 1$, but disagree noticeably for small $N$.

From dimensional analysis it can also be deduced [using Eq. (A10)], that the GW spectrum for MD scales as $\left.h_{0}^{2} \Omega_{\mathrm{GW}}^{(0)}(f) \propto \frac{f_{\mathrm{eq}}^{2}}{f^{2}} F_{\mathrm{MD}}^{(\infty)}\right|_{N \rightarrow \infty}$, where $\left.F_{\mathrm{MD}}^{(\infty)}\right|_{N \rightarrow \infty}$ is a constant that could be obtained from a numerical computation as we did for RD. Numerical integration (with sufficient accuracy) of the analytical spectrum is however costly (due to oscillations of the Bessel functions present in the solution to the self-ordering dynamics; see Appendix A). Furthermore, as mentioned before, from an observational point of view, only the part of the GW spectrum generated during RD is relevant, as only that part of the spectrum is potentially observable by GW experiments like PTAs, or present and planned direct-detection GW interferometers. Since we have already clarified the overall shape of the GW spectrum over all frequencies, in the remainder of the paper we will only focus on the RD case.

\section{LATTICE COMPUTATION OF GWs. PART 1: UNEQUAL-TIME CORRELATORS}

Now we present our numerical results, based on lattice simulations. We use two different numerical methods to obtain the energy density spectrum of the GWs emitted by the network of cosmic defects. In this section, we discuss our results from lattice-based UETCs of the defects' energy-momentum tensor, which serve as an input to compute the GW spectrum.

Our starting point for a lattice simulation of global defects is to consider a scalar field $\Phi$ with $N$ (real) components $\Phi=\left(\phi_{1}, \phi_{2}, \ldots, \phi_{N}\right)^{\mathrm{T}} / \sqrt{2}$, and a potential $V(\Phi)=$ $\lambda\left(|\Phi|^{2}-v^{2} / 2\right)^{2}$, where $\lambda$ is the dimensionless selfcoupling, $v$ is the VEV in the broken phase, and $|\Phi|^{2}=\frac{1}{2} \sum_{b} \phi_{b}^{2}$. When due to some interaction with other degrees of freedom (represented either by another field or by a thermal plasma), the tachyonic mass in $V(\Phi)$ 
dominates over other interaction terms, the $O(N)$ symmetry is spontaneously broken to $O(N-1)$. The scalar field reaches a (spatially dependent) expectation value, very close to $|\Phi|=v / \sqrt{2}$ in most regions.

For $N=2$ and $N=3$, the field remains zero along lines and points in space, creating strings and monopoles. The results for these cases are rather different to the ones obtained in the large- $N$ limit considered before, both because of the presence of the topological defects, and because $N$ is not large, meaning that the nonlinear terms in the field equations neglected in the large- $N$ approximation are important. As for higher $N$ no topological defects are formed, the numerical results should approach the analytic ones in the large- $N$ limit. The case $N=4$ is a boundary case. Here, the field can leave the vacuum manifold, but only at isolated spacetime points, in field configurations called textures. The difference between the linear and nonlinear sigma model dynamics turns out to be minimal $[136,137]$.

In the following we explain the procedure to obtain the GW spectrum from a model with a global $O(N)$ symmetry, solving the EOM of the system and obtaining the UETCs corresponding to the system. We simulate cases ranging from small values of $N$, to cases closer and closer to the large- $N$ limit; in particular we consider $N=2,3,4,8,12$, 20. The EOMs for the field components are, in the continuum,

$$
\ddot{\phi}_{b}+2 \mathcal{H} \dot{\phi}_{b}-\vec{\nabla}^{2} \phi_{b}=-2 \lambda a^{2}\left(|\Phi|^{2}-v^{2} / 2\right) \phi_{b},
$$

with $b=1, \ldots, N$, and where we have kept $\lambda$ as a parameter. The lattice version of this equation can be found in Appendix B. Here it is relevant to note that due to the presence of the factor $a^{2}$ on the rhs of Eq. (47), the size of the defects, say the widths of strings or the radii of monopoles, shrink in comoving coordinates, as the system evolves. This is a well-known and well-studied issue in lattice simulations of defect networks. As in previous studies in the literature, we will use the Press-RydenSpergel (PRS) method [138] to deal with this problem. This amounts to keeping $a^{2} \lambda=\lambda_{c}=$ const, so that the physical scalar mass parameter shrinks as $m_{\Phi}=\sqrt{\lambda} v \propto 1 / a$, and hence the width of the topological defects grows linearly with the scale factor $\delta l \sim 1 / m_{\Phi} \propto a(t)$.

The algorithm we use in our numerical simulations, solves the lattice version of the EOMs (47) on a periodic Cartesian grid, using a seven-point stencil for the threedimensional Laplacian and a leapfrog scheme for the time evolution. As mentioned above, we keep a constant comoving scalar mass $m_{c}=\sqrt{\lambda_{c}} v=a m_{\Phi}$. Space and time coordinates $x^{\mu}$ are measured in terms $m_{c}^{-1}$ units, whereas field scalar amplitudes are in units of $v$, and field derivatives are in units of $m_{c} v$. The grid size $N^{3}$ of our simulations varies from
$1024^{3}$ to $2048^{3}$, with lattice spacings $\Delta x=0.5 m_{c}^{-1}-$ $1.0 m_{c}^{-1}$ and time step $\Delta t=0.2 \Delta x$.

In our simulations, we are not interested in the initial configuration of the fields; what we are after is the scaling regime. Once scaling is reached, the memory of the initial configuration is gone, so the only importance of the initial configuration is that it should lead the system to scaling as fast as possible. We initialize the fields with independent random values constrained to lie on the vacuum manifold, i.e., within a $(N-1)$-sphere $\Phi^{\mathrm{T}} \Phi=v^{2} / 2$, and with $\dot{\Phi}=0$. After an initial transient time with diffusion evolution, we start evolving the fields with the lattice version of the EOMs (47) (see Appendix B) and eventually the system relaxes into the scaling regime. In practice we enforce that the diffusion phase lasts for as long as it takes to reach the condition $\left(1 / 2-|\Phi|^{2} / v^{2}\right)^{1 / 2} \leq 0.01$, which takes typically a time of the order of $t_{0.01} \approx 30 \mathrm{~m}_{c}^{-1}$ for $N>3$ and $t_{0.01} \approx$ $50 m_{c}^{-1}$ for $N=2$, 3. In order to determine the time when the network reaches scaling, $t_{\text {sca }}$, we find the time at which the correlation-length estimator based on the energy density of the system begins to be linearly proportional to time [139]. We also complement this method by tracking the overlapping of the energy-momentum correlators (see below). For most cases, e.g., in $\mathrm{RD}$, the system reaches scaling at a time $t_{\mathrm{sca}} \approx 80 m_{c}^{-1}+t_{0.01}$, except for $N=2$, where scaling is reached later, $t_{\mathrm{sca}} \approx 130 m_{c}^{-1}+t_{0.01}$.

\section{A. Unequal-time correlators}

The energy-momentum tensor for the model is given by

$$
T_{\mu \nu}(\mathbf{x}, t)=2 \partial_{\mu} \Phi^{\mathrm{T}} \partial_{\nu} \Phi+g_{\mu \nu} \mathcal{L}(\Phi) .
$$

The method of calculating UETCs from classical lattice field theory simulations is well documented [128,133,140,141], and we will just briefly summarize it here.

The only UETCs we are interested in this work are the tensor UETCs since those are the ones contributing to the GW. Taking the spatial Fourier transform of $T_{i j}$, the two tensor polarizations $(A=1,2)$ are given by

$$
S_{A}^{T}(\mathbf{k}, t)=\sqrt{\frac{t}{2}} \sum_{i, j} M_{i j}^{A} T_{i j}(\mathbf{k}, t),
$$

where the projectors $M_{i j}^{A}$ are defined as

$$
\begin{aligned}
& M_{i j}^{1}=\frac{1}{2}\left(e_{i}^{1} e_{j}^{2}+e_{i}^{2} e_{j}^{1}\right), \\
& M_{i j}^{2}=\frac{1}{2}\left(e_{i}^{1} e_{j}^{1}-e_{i}^{2} e_{j}^{2}\right),
\end{aligned}
$$

in the vector basis where $k_{i} e_{i}^{A} e_{j}^{B}=0$ and $\delta_{i j} e_{i}^{A} e_{j}^{B}=\delta^{A B}$. They obey 


$$
\sum_{A} M_{i j}^{A} M_{l m}^{A}=\Lambda_{i j, l m}
$$

where $\Lambda_{i j, l m}$ is the projector onto the TT part of a tensor, defined in Eq. (7). The UETC is obtained as

$$
\mathcal{U}\left(x_{1}, x_{2}\right)=\frac{1}{2} \sum_{A}\left\langle S_{A}^{T}\left(\mathbf{k}, t_{1}\right) S_{A}^{T}\left(\mathbf{k}, t_{2}\right)^{*}\right\rangle,
$$

where the average is taken over a set of numerical simulations and a shell in Fourier space. Note that the UETC obeys the symmetry $\mathcal{U}\left(x_{1}, x_{2}\right)=\mathcal{U}\left(x_{2}, x_{1}\right)$.

We only compute the corresponding UETCs once the system has reached scaling. We construct them by multiplying the Fourier transforms of the TT-projected energymomentum tensors at equally spaced times $t_{\text {ref }}<t_{1}<t_{\text {end }}$, $\Delta t=10$ for this work. We made a conservative choice for the UETC extraction initialization time- $t_{\text {ref }}=128$ for all cases except for $N=2$ where $t_{\text {ref }}=200$-while we respect the half-box light crossing time for the last UETC extraction, i.e., $t_{\text {end }}=L / 2$, where $L=1024$ for $N=2,3,4$ and $8, L=768$ for $N=12$ and $L=512$ for $N=20$. In order to obtain the power spectra we average over a shell of width $\Delta k=2 \pi / L$, where $L$ is the side length of the simulation volume.

In Fig. 2 we show the comparison between the equaltime correlators (ETCs) from our numerical method above, denoted $E_{\text {num }}(x)$, with the one obtained in the large- $N$ limit analytical calculation, denoted $E_{\mathrm{th}}$. The numerically obtained ETCs are the average over five different simulations. In the figure, we multiply the ETCs by $N$, as the output from the theory is the value of $N E_{\text {th }}(x)$ as $N \rightarrow \infty$. We observe that the discrepancies are larger at higher $x=$ $k t$ than at lower $x$, whereas the discrepancy is reduced the

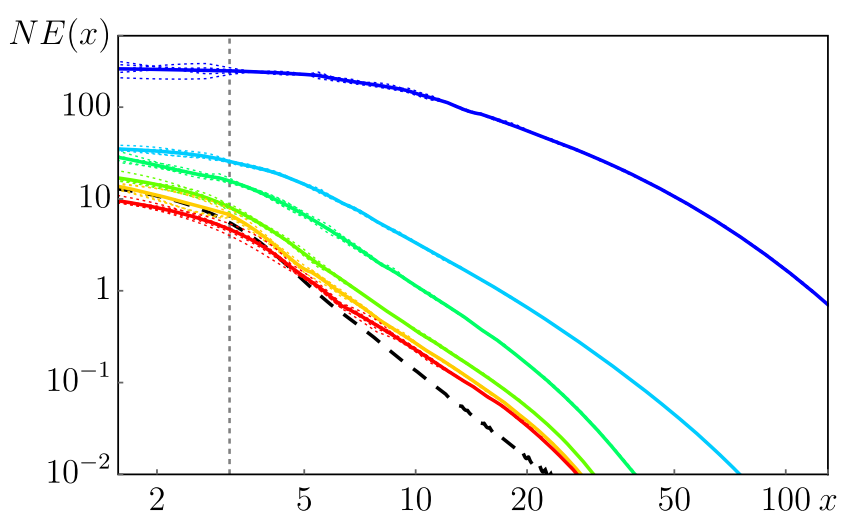

FIG. 2. Comparison between the ETCs from numerical simulations $E_{\text {num }}(x)$ (color gradient) and theoretical analytical large- $N$ expressions (black dashed) $E_{\mathrm{th}}(x)$. All ETCs are obtained at the reference time. The colored lines correspond to (from top to bottom) $N=2,3,4,8,12$ and 20. All five realizations have been included, where each dotted line corresponds to individual runs and solid lines to the mean ETC. A dashed vertical line at $x=\pi$ is also included to show the point where $\Upsilon_{N}$ is computed. larger the value of $N$. We will therefore discuss the cases $N=2$ and $N=3$ separately.

(1) $N \geq 4$. The figure shows that the ETCs for the $N=4,8,12$ and 20 cases are close to the theoretical large- $N$ prediction. The larger the $N$, the closer the ETC approaches $E_{\text {th }}$ (this effect is more evident at small $x$, i.e., at larger length scales).

(2) $N=2$ (strings) and $N=3$ (monopoles). These two cases have the lowest value of $N$, and hence are the ones expected to be the furthest from the analytical prediction, because 1) the field evolution is not linear, and 2) the vacuum manifold has nontrivial topology, and hence topological defects are expected to form. For the $O(2)$ case, the homotopy group of the vacuum is $\pi_{1} \neq 1$ and thus the defects are (global) cosmic strings, whose dynamics are rather different to the dynamics of nontopological field configurations. In particular, the time needed to reach scaling is larger, which makes the extraction of the scaling UETCs more complicated. In the $O(3)$ case, the topology of the vacuum is also nontrivial $\left(\pi_{2} \neq 1\right)$, and global monopoles are formed in this model $[139,142,143]$. The departure from the large$N$ limit is however not as extreme as in $O(2)$. As we will see, the spectral amplitude of the GW background $\Omega_{\mathrm{GW}}$ deviates with respect to the large- $N$ analytical computation by factors of $\mathcal{O}(100)$ and $\mathcal{O}(10)$, when evaluated at $N=2$ and 3, respectively.

In Sec. IV B, we will quantify the discrepancy of the numerical correlators against the analytical computation in the large- $N$ limit, and in particular how this impacts the GW signal.

\section{B. Calculation of the GW signal}

As indicated by Eq. (25), there is a direct connection between the GW energy density spectrum $\Omega_{\mathrm{GW}}$ and the UETC $\mathcal{U}\left(x_{1}, x_{2}\right)$ for each value of $N$,

$$
\Omega_{\mathrm{GW}} \propto \int d x_{1} d x_{2} \frac{a_{1} a_{2}}{\sqrt{x_{1} x_{2}}} \cos \left(x_{1}-x_{2}\right) \mathcal{U}\left(x_{1}, x_{2}\right),
$$

where $a_{1} \equiv a\left(x_{1} / k\right), a_{2} \equiv a\left(x_{2} / k\right)$. Numerically obtained UETCs from lattice simulations can be used to obtain the GW energy density power spectrum for each case, by a simple two-dimensional numerical integration. The specific formula for RD is given by Eq. (30), whereas for MD it is given by Eqs. (32) and (33). In the following, we will use these formulas, and in particular their redshifted versions (31) and (34), to numerically calculate the spectrum of the GWs emitted by a network of global defects in the $O(N)$ model. We will compare these numerical results (in particular for the RD case) with the analytical prediction based on the large- $N$ computation.

As observed in Sec. IV A, the smaller the $N$, the more the numerical UETCs deviate from the analytical prediction. 
A simple way to quantify this difference is to compare the ETC $E(x)=\mathcal{U}(x, x)$ for each value $N$. In particular, we define

$$
\Upsilon_{N} \equiv \frac{E_{\mathrm{num}}(\pi)}{E_{\mathrm{th}}(\pi)}
$$

as the ratio between the numerical and theoretical computations of ETCs, evaluated at a scale $x=\pi$, corresponding to the moment when half a wavelength has entered the horizon at RD. The values we find for $\Upsilon$ are shown in Table I. As expected, the numerical ETC approaches the theoretical prediction as $N$ grows, as indicated by the approach of $\Upsilon_{N}$ to unity as $N$ increases. For the case of cosmic strings $(N=2)$, the numerical ETC is a factor $\sim 100$ bigger than the analytical one, signaling a complete breakdown of the large- $N$ approximation.

We focus on the GWs produced during $\mathrm{RD}$, as this is the relevant part of the spectrum for direct-detection experiments. In order to numerically obtain the GW spectrum, we use the lattice version of Eq. (31)

$$
\left.h_{0}^{2} \Omega_{\mathrm{GW}}^{\mathrm{num}}\right|_{\mathrm{RD}}=h_{0}^{2} \Omega_{\mathrm{rad}}^{(0)}\left(\frac{v}{M_{\mathrm{Pl}}}\right)^{4} F_{\mathrm{RD}}^{(\mathrm{num})},
$$

with

$$
\begin{aligned}
F_{\mathrm{RD}}^{(\mathrm{num})} \equiv & \frac{64}{3} \int_{x_{\min }}^{x_{\max }} d x_{1} \int_{x_{\min }}^{x_{\max }} d x_{2} \sqrt{x_{1} x_{2}} \\
& \times \cos \left(x_{1}-x_{2}\right) \mathcal{U}\left(x_{1}, x_{2}\right) .
\end{aligned}
$$

The $\mathcal{U}\left(x_{1}, x_{2}\right)$ are the numerical UETCs during $\mathrm{RD}$, obtained for each value of $N$, and as explained before, $F_{\mathrm{RD}}^{(\mathrm{num})}$ is the only quantity we need to extract from the simulations. Even though in a lattice we are always bounded by IR and UV scales (due to the finite volume and lattice spacing of the grid), we have made sure that $F_{\mathrm{RD}}^{(\mathrm{num})}$ only changes marginally, whenever we slightly

TABLE I. Values of the numerical ETCs at $x=\pi$, and GW amplitudes today, normalized to the large- $N$ calculation. Top table: ratios from simulations in this paper. Bottom table: ratios taken from Paper I. The statistical fluctuations are less than $10 \%$ in both cases.

\begin{tabular}{lcccccc}
\hline \hline \multicolumn{7}{c}{ Current data } \\
\hline$N$ & 2 & 3 & 4 & 8 & 12 & 20 \\
$\Upsilon_{N}$ & 45 & 4.6 & 2.8 & 1.5 & 1.2 & 0.9 \\
$\Sigma_{N}$ & 238 & 10 & 4.1 & 1.7 & 1.3 & 1.0 \\
\hline \hline
\end{tabular}

\begin{tabular}{lcccccc}
\hline \hline \multicolumn{7}{c}{ From Paper I } \\
\hline$N$ & 2 & 3 & 4 & 8 & 12 & 20 \\
$\Upsilon_{N}$ & 36 & 4.5 & 3.1 & 1.7 & 1.4 & 1.3 \\
$\Sigma_{N}$ & 130 & 7.3 & 3.9 & 1.8 & 1.4 & 1.3 \\
\hline \hline
\end{tabular}

change the boundary values $x_{\min }, x_{\max }$, or change the number of points per dimension $N_{p}$. In all our simulations we see that $F_{\mathrm{RD}}^{(\mathrm{num})}$ converges rapidly and asymptotically for $x_{\max } \gg 1$ to the constant value $F_{\mathrm{RD}}^{(\infty)}$. Therefore, from our simulations we can obtain $F_{\mathrm{RD}}^{(\mathrm{num})} \approx F_{\mathrm{RD}}^{(\infty)}$.

All in all, we computed $\mathcal{U}\left(x_{1}, x_{2}\right)$ from our numerical simulations, which in turn can be turned into $F_{\mathrm{RD}}^{(\mathrm{num})} \approx F_{\mathrm{RD}}^{(\infty)}$, and this can be substituted into Eq. (56) to obtain the numerical GW spectrum $\left.\Omega_{\mathrm{GW}}^{\text {num }}\right|_{\mathrm{RD}}$.

In Table I, we also provide the lists of ratios of the asymptotic amplitudes of the $\mathrm{GW}$ spectrum during $\mathrm{RD}$, comparing the lattice result to the theoretical amplitude obtained in the large- $N$ limit,

$$
\Sigma_{N}=\frac{\Omega_{\mathrm{GW}}^{\mathrm{num}}}{\Omega_{\mathrm{GW}}^{N \rightarrow \infty}},
$$

for different values of $N$.

If we fit the new numerical amplitudes against $N$, we obtain

$$
\left.\Omega_{\mathrm{GW}}^{\mathrm{num}}\right|_{\mathrm{RD}}=\left.\Omega_{\mathrm{GW}}^{N \rightarrow \infty}\right|_{\mathrm{RD}}\left(a_{0}+\frac{a_{2}}{N^{2}}+\cdots\right)
$$

with $a_{0}=0.91 \pm 0.11$ and $a_{2}=51.1 \pm 3.5$, and we obtain a negligible value for $a_{1}$. The above formula is valid only for $N \geq 4$. The fit shows evidence that the numerical results converge to the large- $N$ calculation as $N^{-2}$, albeit with a large coefficient, confirming the result of Paper I.

The comparison between the results obtained in Paper I and the current work can be observed in Table I. Note that the statistical fluctuations between simulations are less than 10\%. Also, Fig. 3 shows the comparison, where the standard deviations around the mean are depicted by the vertical lines. It can be seen that the numbers obtained in Paper I and in the present work agree rather well and are

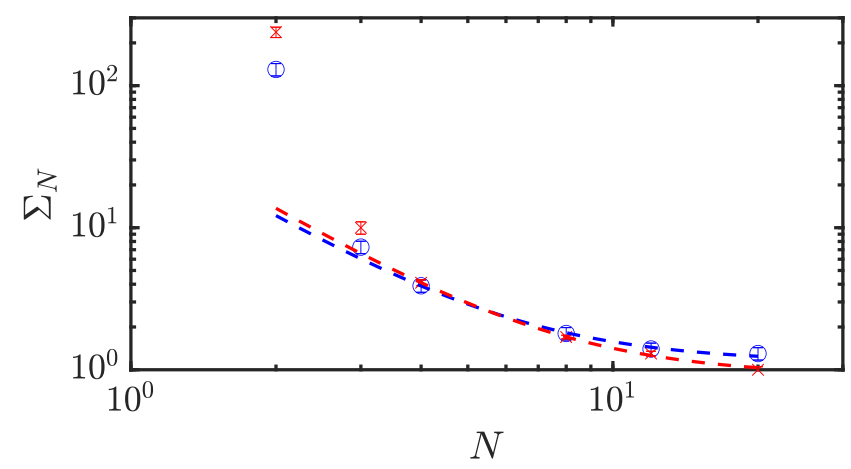

FIG. 3. $\Sigma_{N}$ from Table I and the comparison of the fit (59) for $N \geq 4$ from Paper I (blue circles) and the current work (red crosses), including statistical uncertainties. Data for $N=2$ and $N=3$ have also been included for completeness. 
consistent (except for the $N=2$ case, which we explain separately).

Turning to $N=2$, we can see in Table I that the value of $\Sigma_{2}$ has increased by roughly a factor of 2 . As mentioned earlier, this case is special because global cosmic strings are formed. It takes longer to reach scaling than for other cases, and therefore the time the network is simulated in scaling is shorter. Moreover, while the network length scale grows linearly in time, the intercept of the line with the $t$ axis is offset from zero, as explained in Refs. [87,123,128,130,133]. This time offset is fed into the definition of the UETCs [note the factor of $\sqrt{t}$ in Eq. (49)], and therefore makes the UETC (and therefore the GW signal) larger. The fact that the value of $\Sigma_{2}$ has almost doubled can be accounted for by the value of the time offset in the simulations: while time offset was not considered for the values reported in Paper I, it is included in the computation of $\Sigma_{2}$ of this work. Taking this time offset into account in our old simulations reported in Ref. [117], the numbers become closer. Some differences are also to be expected because of the larger volume in this work. Further investigation is needed to understand and reduce the uncertainties in our measurement of $\Sigma_{2}$.

\section{Comparison with eigenvector decomposition}

A standard approach for computations of CMB fluctuations from topological defects is the decomposition of the UETCs into a basis of its eigenvectors by diagonalization, and then summing the power spectra resulting from each eigenvector, appropriately weighted by its eigenvalue. This technique can also be applied to the GW power spectrum calculation. In this section, we check the convergence of the partial sums over a series of weighted eigenvector/eigenvalue terms to the GW energy density power spectrum obtained directly from the UETC.

Our UETC is naturally discretized in $N_{s}=2048$ steps in each of the scaled wave number variables $x, x^{\prime}$, so we are dealing with $N_{s} \times N_{s}$ symmetric positive matrices. We can then diagonalize them, finding an orthonormal base of eigenvectors $\left\{v_{n}(x)\right\}$, with real positive eigenvalues $\lambda_{i}>0$ that can be ordered as $\lambda_{1}>\lambda_{2}>\lambda_{3}>\ldots 0$. Every UETC can then be written as

$$
\mathcal{U}\left(x_{1}, x_{2}\right)=\sum_{n} \lambda_{n} v_{n}\left(x_{1}\right) v_{n}^{*}\left(x_{2}\right)
$$

with the eigenvalues such that $0<\lambda_{n+1}<\lambda_{n}$. This can be applied to both UETCs from RD and MD. In the case of RD, Eq. (60) allows $F_{\mathrm{RD}}^{(\mathrm{num})}$ to be decomposed as

$$
F_{\mathrm{RD}}^{(\mathrm{num})}=\sum_{n} \lambda_{n}\left\{\left|\mathcal{S}_{\mathrm{RD}}^{(\mathrm{n})}\right|^{2}+\left|\mathcal{C}_{\mathrm{RD}}^{(\mathrm{n})}\right|^{2}\right\}
$$

with

$$
\begin{aligned}
\mathcal{S}_{\mathrm{RD}}^{(\mathrm{n})} & \equiv \frac{8}{\sqrt{3}} \int_{x_{\text {min }}}^{x_{\max }} d x x^{1 / 2} \sin (x) v_{n}(x), \\
\mathcal{C}_{\mathrm{RD}}^{(\mathrm{n})} & \equiv \frac{8}{\sqrt{3}} \int_{x_{\min }}^{x_{\max }} d x x^{1 / 2} \cos (x) v_{n}(x) .
\end{aligned}
$$

This leads to

$$
\begin{aligned}
\left.\Omega_{\mathrm{GW}}^{\mathrm{num}}\right|_{\mathrm{RD}} & =\left.\sum_{n} \Omega_{\mathrm{GW}}^{(\mathrm{n})}\right|_{\mathrm{RD}} \\
& \equiv \sum_{n} \lambda_{n} \Omega_{\mathrm{rad}}^{(0)}\left(\frac{v}{M_{\mathrm{Pl}}}\right)^{4}\left\{\left|\mathcal{S}_{\mathrm{RD}}^{(\mathrm{n})}\right|^{2}+\left|\mathcal{C}_{\mathrm{RD}}^{(\mathrm{n})}\right|^{2}\right\} .
\end{aligned}
$$

For MD, an equivalent expression can be written as

$$
\begin{aligned}
\left.\Omega_{\mathrm{GW}}^{\mathrm{num}}\right|_{\mathrm{MD}} & =\left.\sum_{n} \Omega_{\mathrm{GW}}^{(\mathrm{n})}\right|_{\mathrm{MD}} \\
& \equiv \sum_{n} \lambda_{n} \Omega_{\mathrm{rad}}^{(0)}\left(\frac{v}{M_{\mathrm{Pl}}}\right)^{4} \frac{k_{\mathrm{eq}}^{2}}{k^{2}}\left\{\left|\mathcal{S}_{\mathrm{MD}}^{(\mathrm{n})}\right|^{2}+\left|\mathcal{C}_{\mathrm{MD}}^{(\mathrm{n})}\right|^{2}\right\},
\end{aligned}
$$

where $\mathcal{S}_{\mathrm{MD}}^{(\mathrm{n})}, \mathcal{C}_{\mathrm{MD}}^{(\mathrm{n})}$ are analogous expressions to Eqs. (62)(63), but substituting $\frac{8}{\sqrt{3}} \rightarrow \frac{8}{\sqrt{3}}(\sqrt{2}-1)$ and $x^{1 / 2} \rightarrow x^{3 / 2}$ (inside the integrals).
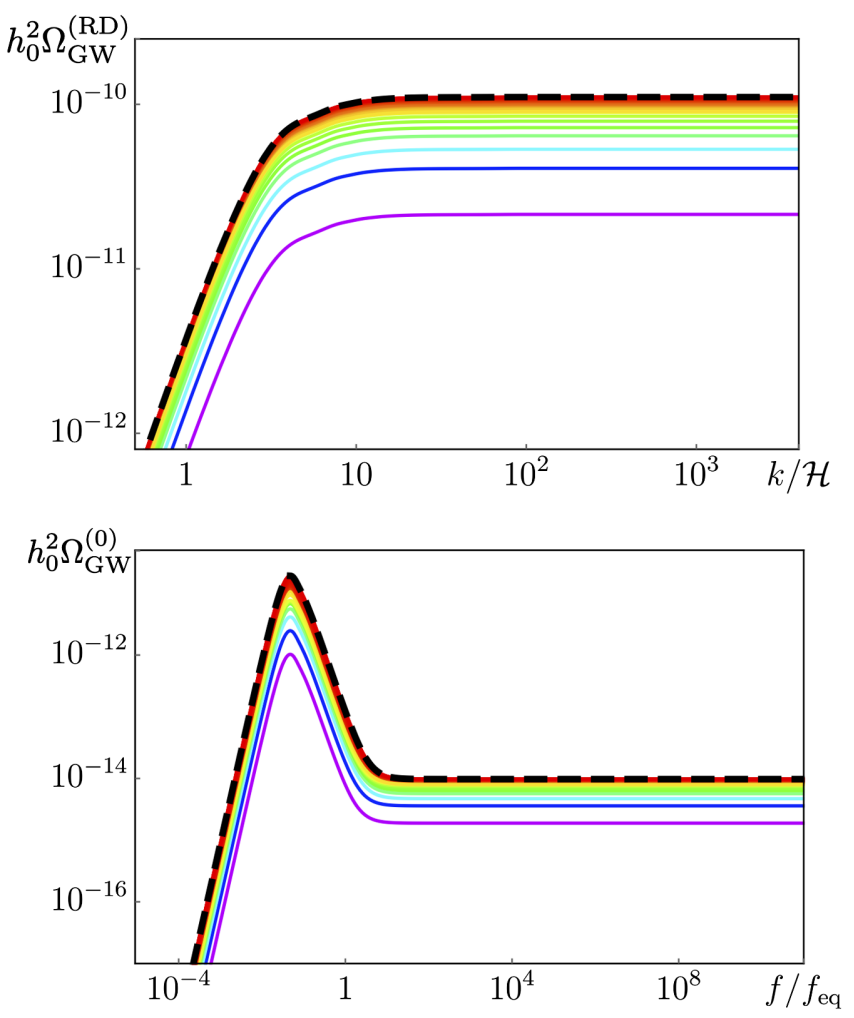

FIG. 4. Example of the GW background reconstruction from eigenvectors and eigenvalues in the $O(4)$ model during RD (top panel, with $\mathcal{R}_{*} / \mathcal{R}_{t}=1$ ) and today's full redshifted spectrum (lower panel, with $\mathcal{R}_{*}=1$ ). The signals obtained from the full UETC are shown with black dashed lines. 
In Fig. 4 we show the reconstruction of the GW spectrum for the $O(4)$ model during $\mathrm{RD}$, as well as of today's full redshifted spectrum. Each line represents a spectrum reconstructed with the contribution of one more eigenfunction added, so the bottom line corresponds to having considered only the first term $n=1$, the next line above corresponds to having summed the first two terms $n=1,2$, and so on, all they way up to the highest line, which represents the sum of all the terms. The signal from direct integration of the UETC is indicated with dashed lines.

As each term we add in the series is weighted by successively smaller eigenvalues, newer contributions contribute less and less. We observe that after adding only $\sim 15$ terms, the spectrum is already reconstructed to better than $\sim 10 \%$.

\section{LATTICE COMPUTATION OF GWs. PART 2: TENSOR REAL-TIME EVOLUTION}

Let us consider now the relativistic wave equation introduced in Sec. II, that governs the dynamics of GWs

$\ddot{h}_{i j}(\mathbf{x}, t)+2 \mathcal{H} \dot{h}_{i j}(\mathbf{x}, t)-\nabla^{2} h_{i j}(\mathbf{x}, t)=\frac{16 \pi}{M_{\mathrm{Pl}}^{2}} \Pi_{i j}^{\mathrm{TT}}(\mathbf{x}, t)$,

with dots denoting derivatives with respect to the conformal time. In our case, the TT part of the anisotropic stress tensor $\Pi_{i j}^{\mathrm{TT}}$ - the source of the GWs-is given by

$$
\Pi_{i j}^{\mathrm{TT}}(\mathbf{x}, t) \equiv \sum_{b}\left(\partial_{i} \phi_{b} \partial_{j} \phi_{b}\right)^{\mathrm{TT}},
$$

where $\left\{\phi_{b}\right\}$ are the $N$ components of the scalar field $\Phi=\left(\phi_{1}, \phi_{2}, \ldots, \phi_{N}\right)^{\mathrm{T}} / \sqrt{2}$. For convenience, let us relabel the tensor perturbation as

$$
h_{i j}(k, t) \equiv 16 \pi\left(\frac{v}{M_{\mathrm{Pl}}}\right)^{2} w_{i j}(k, t),
$$

so that their EOM can be written in terms of the dimensionless field variables $\tilde{\phi}_{a} \equiv \phi_{c} / v$, as

$$
\ddot{w}_{i j}+2 \mathcal{H} \dot{w}_{i j}-\nabla^{2} w_{i j}=\sum_{b}\left(\partial_{b} \tilde{\phi} \partial_{b} \tilde{\phi}_{b}\right)^{\mathrm{TT}} .
$$

The spectrum of the GW energy density contained within a volume $V$ [Eq. (12)] can be written in terms of the $w_{i j}$ variables as

$\frac{d \rho_{\mathrm{GW}}}{d \log k}(k, t)=\frac{4 k^{3} v^{4}}{\pi a^{2}(t) M_{\mathrm{Pl}}^{2} L^{3}}\left\langle\dot{w}_{i j}(k, t) \dot{w}_{i j}(k, t)\right\rangle_{4 \pi}$,

where we have introduced $V=L^{3}$, where $L$ is the side length of the lattice, and defined $\langle\ldots\rangle_{4 \pi} \equiv \frac{1}{4 \pi} \int d \Omega_{k} \ldots$, where $d \Omega_{k}$ is a solid angle differential in $\mathbf{k}$ space.
In order to numerically solve the EOM for the GWs on the lattice, Eq. (69), we have followed the procedure originally introduced in Ref. [43]. We solve (a lattice version of) a relativistic wave equation for an unphysical perturbation $u_{i j}$

$$
\ddot{u}_{i j}+2 \mathcal{H} \dot{u}_{i j}-\nabla^{2} u_{i j}=\sum_{b}\left(\partial_{i} \tilde{\phi}_{b} \partial_{j} \tilde{\phi}_{b}\right),
$$

with no TT projection over the source. We can then recover the physical TT part $w_{i j}$ at any moment through

$$
w_{i j}(k, t)=\Lambda_{i j, l m}(\hat{k}) u_{l m}(k, t),
$$

where $\Lambda_{i j, l m}(\hat{k})$ is the transverse-traceless projector (6). Since $\Lambda_{i j, p q}(\hat{k}) \Lambda_{p q, l m}(\hat{k})=\Lambda_{i j, l m}(\hat{k})$, the argument inside the angular average $\langle\ldots\rangle$ in Eq. (70), can be computed as

$$
\dot{w}_{i j}(k, t) \dot{w}_{i j}(k, t)=\dot{u}_{i j}(k, t) \Lambda_{i j, l m}(\hat{k}) \dot{u}_{l m}(k, t) .
$$

Appendix C explains this procedure of obtaining GWs in a lattice.

We have studied the real-time GW generation process for a model with $N=4$ scalar fields, in lattices up to $N=$ 2048 points per dimension. To solve the scalar field dynamics we have used the same standard lattice formulation as in Sec. IV. In all simulations we have ensured that the lattice resolution covers well the dynamical range of momenta excited in the process, for both the scalar fields and the GWs; see e.g., the discussion in Sec. V C.

Defining $d \tilde{x}=m_{\mathrm{c}} d x$ as the dimensionless lattice spacing, with $m_{\mathrm{c}} \equiv \sqrt{\lambda_{c}} v$ and $\lambda_{c}=a^{2} \lambda=$ const, so that $z=$ $m_{c} t$ and ${ }^{\prime} \equiv \partial / \partial z$, the final expression of the GW spectrum on the lattice reads

$$
\begin{aligned}
\frac{d \rho_{\mathrm{GW}}}{d \log k}(\tilde{\mathbf{n}}, z)= & \frac{4}{\pi}\left(\frac{v}{M_{\mathrm{Pl}}}\right)^{4} \frac{d \tilde{x}^{3} \kappa(\tilde{\mathbf{n}})^{3}}{N^{3}} \frac{\lambda_{c} v^{2} M_{\mathrm{Pl}}^{2}}{a(z)^{4}} \\
& \times a^{2}(z)\left\langle u_{i j}^{\prime}(\tilde{\mathbf{n}}, z) \Lambda_{i j, l m}^{(\mathrm{L})}(\hat{k}) u_{i j}^{\prime}(\tilde{\mathbf{n}}, z)\right\rangle_{4 \pi},
\end{aligned}
$$

where $\Lambda_{i j, l m}^{(\mathrm{L})}(\hat{k})$ is the lattice TT projector, $\kappa(\tilde{\mathbf{n}}) \equiv k(\tilde{\mathbf{n}}) / m_{c}$ is the dimensionless lattice momenta, where $k(\tilde{\mathbf{n}}) \equiv k_{\mathrm{IR}}|\tilde{\mathbf{n}}|$ is the momentum at the Fourier lattice site $\tilde{\mathbf{n}}=\left(\tilde{n}_{1}, \tilde{n}_{2}, \tilde{n}_{2}\right)$, $-\frac{N}{2}+1 \leq \tilde{n}_{j} \leq \frac{N}{2}, k_{\mathrm{IR}}=\frac{2 \pi}{L}$ is the minimum lattice momentum, and $w_{i j} \equiv w_{i j}(\tilde{\mathbf{n}}, t)$ is the discrete Fourier transform of $w_{i j}(\mathbf{n}, z)$, where $\mathbf{n}=\left(n_{1}, n_{2}, n_{3}\right)$ indicates the lattice sites, and $0 \leq n_{j} \leq N-1$.

In practice, on the lattice we solve the discretized version of the scalar fields' EOM (47) living in a background of RD with $a(z) \propto z$, together with Eq. (71) for the (unphysical) spatial metric perturbations. We obtain, via Eq. (74), the physical GW energy density spectrum at any time of the evolution. As explained at the beginning of Sec. IV, after 
setting up the initial random condition for the scalar field components, we evolve the system diffusively, until the scalar field expectation value reaches a small deviation (we choose 1\%) with respect to the true VEV, $\left|\sum_{b} \tilde{\phi}_{b}^{2}-1\right|^{1 / 2} \leq 0.01$, denoting this time by $z_{0.01}$. Once this condition is reached, the scalar field is allowed to follow its PRS-approximated equation of motion (47).

\section{A. Emergence of the GW plateau}

We start the evolution of the EOM (71) for the GWs at a time $z_{\mathrm{GW}} \geq z_{0.01}$, choosing the initial condition $u_{i j}\left(\mathbf{x}, z_{\mathrm{GW}}\right)=\dot{u}_{i j}\left(\mathbf{x}, z_{\mathrm{GW}}\right)=0$ at that moment. Solving the time evolution of the tensor perturbations leads then to a time-dependent GW energy density spectrum, shown in Fig. 5 for a numerical simulation with $N_{p}=2048$, and $d \tilde{x}=1$.

Initially, the spectrum is peaked at a scale of the order of $\sim \mathcal{O}(0.1) m_{c}^{-1}$. The amplitude of the GW spectrum then grows rapidly, and its shape changes, so that the initially suppressed IR part of the bump flattens out as time goes by. After some time, the GW energy density spectrum clearly exhibits a plateau in the IR, at least in the first nine wave number bins.

In Fig. 6, we show the growth of $\Omega_{\mathrm{GW}}(k, t)$ for those wave numbers $k$. One sees that they saturate to a constant value after $k$ becomes subhorizon, for $k t \gtrsim 10$. The most IR bin in our power spectrum does not have time to saturate, which explains why it is slightly below the plateau amplitude reached by the other modes.

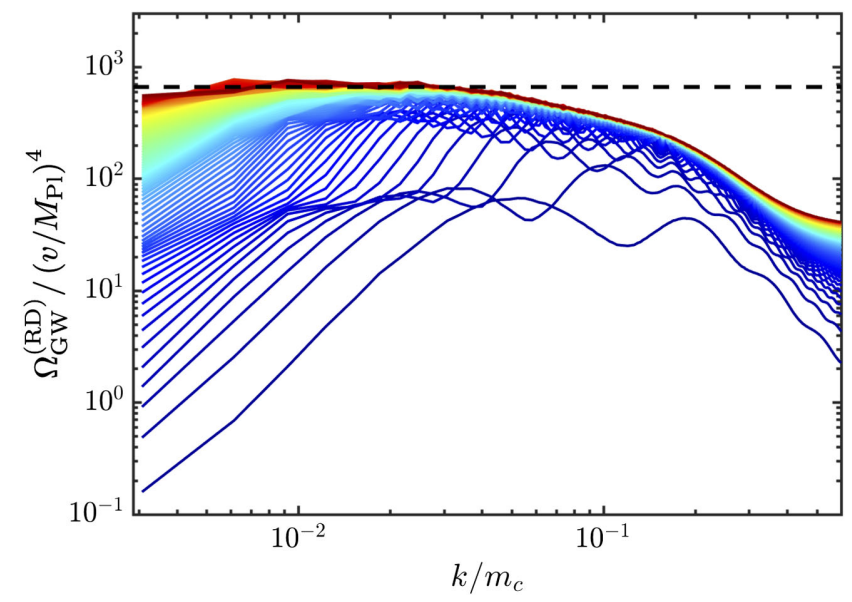

FIG. 5. Real-time evolution of the GW energy density spectrum during $\mathrm{RD}$, for $N_{p}=2048, d \tilde{x}=1$, shown in time intervals $\Delta z=20$, from $z_{\mathrm{RD}}=z_{\mathrm{sca}}=100$ up to $z_{\text {final }}=2000$ (with $\mathcal{R}_{*} / \mathcal{R}_{t}=1$ ). At the most IR scales, from $\kappa=\kappa_{\mathrm{IR}} \simeq 0.3$ up to $\kappa_{*} \simeq 9 k_{\mathrm{IR}} \simeq 3$, we see how the expected plateau gradually forms as time goes by. The plateau is actually well settled once the modes are well inside the horizon (at least an order of magnitude); see Fig. 6. The final plateau settles down precisely at the same amplitude predicted by the UETC technique introduced in Sec. V, here indicated by the dashed horizontal line.

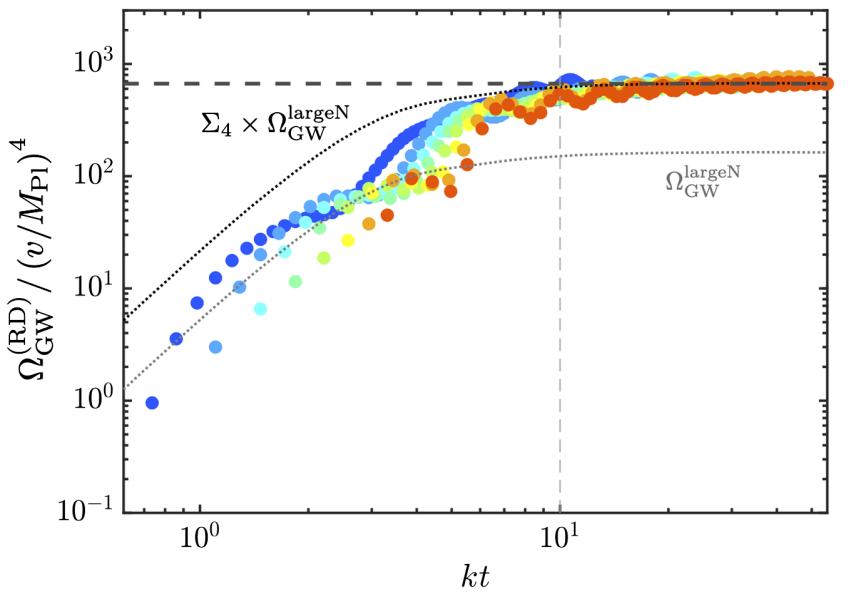

FIG. 6. The evolution of gravitational-wave energy density in selected wave numbers during $\mathrm{RD}$, as a function of $x=k t$, for $N_{p}=2048, d \tilde{x}=1$ (with $\mathcal{R}_{*} / \mathcal{R}_{t}=1$ ). We only plot the spectrum for the nine most IR modes of the simulation, which in Fig. 5 exhibit a plateau shape in the IR. Each mode is represented by a color, starting at blue (lowest $k$ ), passing through green, yellow and orange, and ending at red (highest $k$ relaxing into the plateau). For comparison, we also plot the prediction from the large- $N$ limit of the analytical calculation (lower gray dotted line), also rescaled (higher black dotted line) by the compensating factor $\Sigma_{4}$ to match the UETC lattice results, cf. Eq. (58).

Together, the graphs in Figs. 5 and 7 show how the flat GW spectrum emerges on progressively larger scales, sourced on a scale close to the horizon scale. The growth, and therefore the sourcing, appears to stop for $k t \gtrsim 10$, which is presumably related to the field correlation length,

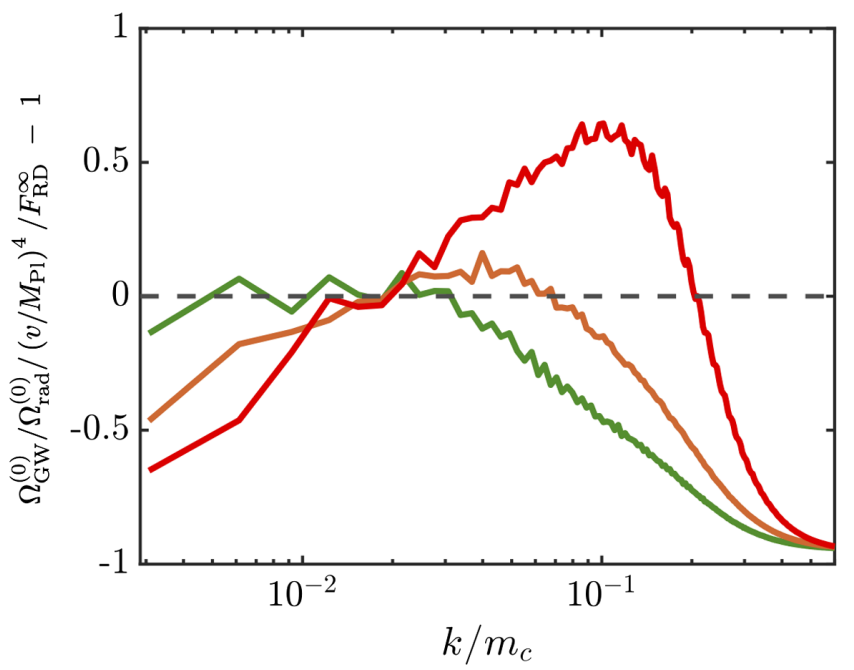

FIG. 7. Relative difference of the real-time evolution GW power spectrum extracted at the end of each simulation (with $\mathcal{R}_{*} / \mathcal{R}_{t}=1$ ), with respect to the plateau calculated from the UETCs $F_{\mathrm{RD}}^{(\infty)}$. All cases are obtained for $\left(N_{p}=2048, d \tilde{x}=1.0\right)$, and correspond to $z_{\mathrm{GW}}^{0}=z_{0.01}=50$ (red), $z_{\mathrm{GW}}=75$ (orange), and $z_{\mathrm{GW}}=z_{\mathrm{sca}}=100$ (green). 
which is slightly less than the horizon scale. We interpret the scale of the initial bump as the initial correlation length of the field.

The behavior of the modes outside the Hubble radius is a useful check of the numerical solution. From the analysis of the superhorizon Green's functions in Sec. III A, the GW spectrum should exhibit an IR tail as $\propto x^{3}$, which implies a $k^{3}$ behavior for the power spectrum, and a $t^{3}$ growth for the individual modes. Both of these expected behaviors are visible in Figs. 5 and 6.

We now compare the GW spectra obtained from the realtime evolution to that obtained from UETCs in Sec. IV. The horizontal line in Fig. 5 represents the GW spectrum $\Omega_{\mathrm{GW}}(k, t)$ calculated via Eq. (30) from the UETCs. Both methods use data from the same simulations. The asymptotic plateau of the real-time GWs agrees very well with the amplitude of the GW spectrum derived from the UETCs.

We also plot the large- $N$ limit of the analytical calculation in Fig. 6, depicted by the dotted gray line, which also exhibits the expected transition from $\propto x^{3}$ at large scales, to $\propto c o n s t$ at smaller scales. If we rescale such an analytical prediction by the compensating factor $\Sigma_{N}$ obtained in Sec. IV for the $N=4$ model [based on the ratio of the $\mathrm{GW}$ spectra obtained from UETCs to the analytical large- $N$ computation, cf. Eq. (58)], the amplitude of the rescaled analytical prediction lies very close to the amplitude obtained for the plateau by the real-time evolution.

The success of the previous comparisons between the UETC-based and real-time GW spectra, provides a consistency check for both methods, and demonstrates that the use of either method should be considered equally acceptable in numerical computations of GWs from scaling seeds. This is one of the most important results of this paper.

\section{B. Importance of scaling for the GW source}

As the scalar field dynamics do not reach a scaling regime until $z=z_{\mathrm{sca}}>z_{0.01}$, a relevant aspect that needs to be quantified is the impact of different choices of $z_{\mathrm{GW}}$ on the GW dynamics.

First of all we should recall that the analytical predictions presented in Sec. III, as well as the numerical computations based on the UETCs obtained in Sec. IV, are based on the scaling regime of the scalar field dynamics. If we switch on the GW evolution when the scalar field dynamics is not yet in scaling, i.e., at some moment $z_{0.01} \leq z_{\mathrm{GW}}<z_{\text {sca }}$, we expect the emerging GW spectrum to differ for each choice of $z_{\mathrm{GW}}$, as the GWs will experience different evolution histories which are not equivalent to each other by a simple "rescaling" of the size of the system into the horizon at each time. In other words, we expect that for GWs switched on too early, the resulting GW spectrum will be unphysical.

In order to check the above phenomena, we performed simulations with several different values of $z_{\mathrm{GW}}$ in the range $z_{0.01} \leq z_{\mathrm{GW}} \leq z_{\mathrm{sca}}$. In Fig. 7 we show the relative difference of GW spectra, extracted at the final simulation time, with respect to the amplitude calculated from the UETCs, obtained when they are switched on at times $z_{\mathrm{GW}}=z_{0.01}+\Delta z_{\mathrm{GW}}$, with $\Delta z_{\mathrm{GW}}=0 \quad$ (red) and 25 (orange), together with the $\mathrm{GW}$ spectra for $z_{\mathrm{GW}}=z_{\text {sca }}$ (green, which corresponds to $\Delta z_{\mathrm{GW}}=50$ ). This figure clearly shows the importance of turning on the GW source when the network is already in scaling. An unphysical bump appears at scales $k / m_{\mathrm{c}} \sim 0.1$ when we start evolving GWs too early, which is nothing but the effect generated by the random initial conditions in the GWs energy density. The defect network is able to forget about the precise nature of its initial field configuration (as scaling implies), but its imprint on the GW energy density spectrum remains and should be avoided. Furthermore, as the figure shows, turning the GW source on too early also fails to create the IR plateau. Hence the plateau is a feature of defect networks which emerges only once a scaling regime is sustained.

\section{Dependence on lattice parameters}

As in any lattice simulation, we cannot choose arbitrarily large volumes (i.e., arbitrarily small $k_{\mathrm{IR}}$ scales), as the UV scales also need to be resolved with sufficient accuracy. We have therefore made sure that in our simulations the amplitude of the GW spectra in the UV scales is well below the amplitude of the plateau in the IR scales (in some cases even exponentially suppressed when the UV coverage is good enough). The GWs emitted at short wavelengths are related to the small scales in the problem, i.e., to the characteristic microscopic scale of the defects $\gtrsim 1 / m_{c}$. The dominant emission of GWs is rather expected due to the dynamics of the whole defect network, dictated by the scaling regime, and hence related to the horizon scale. As the defects self-order themselves around the horizon scale during scaling, GWs are emitted at the horizon scale at each moment of the evolution. We thus need to find a compromise between how well we can cover the IR scales (i.e., how small $k_{\mathrm{IR}}$ can be), and how well we can resolve the microscopic scale $m_{c}^{-1}$ in the UV (i.e., how large a $d x$ we can tolerate, so that we still capture well the defect dynamics). In practice we find that a lattice spacing $d \tilde{x} \equiv m_{c} d x>1$ leads to too large distortions of the UV part of the GW spectrum due to lattice artifacts in the defect dynamics, whereas a lattice spacing $d \tilde{x}<0.5$ leads to a good exponential suppression of the UV tail of the GW spectrum, but only at the expense of the IR coverage, preventing the development of the IR plateau. In practice, we chose $d \tilde{x}=0.5$ and 1.0 and $N_{p}=1024,1512$ and 2048.

We have verified that simulations with different combinations of $\{d \tilde{x}, N\}$, lead to real-time GW power spectra with a very similar amplitude in the overlapping IR region of wave numbers. This can be seen in Fig. 8. Due to intrinsic limitations of our computer resources, the final IR plateau always spans a finite range of momentum scales (in the best case scenario roughly this is around one decade, 


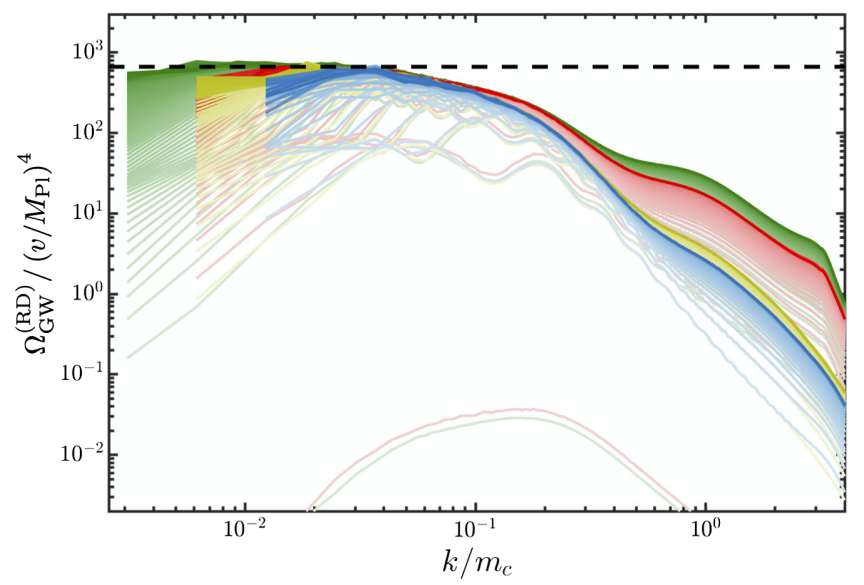

FIG. 8. Example of the real-time evolution of the GW energy density spectrum (with $\mathcal{R}_{*} / \mathcal{R}_{t}=1$ ) for $\left(N_{p}=1024, d \tilde{x}=0.5\right)$ (blue), $\left(N_{p}=1024, d \tilde{x}=1.0\right)($ red $),\left(N_{p}=2048, d \tilde{x}=0.5\right)$ (yellow) and $\left(N_{p}=2048, d \tilde{x}=1.0\right)$ (green).

from the most IR scale $k_{\mathrm{IR}}=2 \pi / L$ of the lattice, up to a scale $\left.k \lesssim 10 k_{\mathrm{IR}}\right)$. The length of the plateau depends on the lattice parameters $\{d \tilde{x}, \tilde{L}\}$. For the largest volume and lattice spacing that we have considered in a simulation $\left(N_{p}=2048, d \tilde{x}=1\right)$, the presence of the IR plateau is clear for around one decade in wave numbers; see e.g., the green curve in Fig. 8. For smaller volumes $(N=1024)$ and/ or lattice spacings $(d \tilde{x}=0.5)$, the plateau is also visible, but over a smaller range of wave numbers. As shown in Fig. 8, the plateau is always present in the overlapping range of wave numbers shared by simulations with different values of $\{d x, \tilde{L}\}$. In particular, the plateau always appears at the same "turn-over" scale, $k_{*} \sim(0.03-0.04) m_{c}$, and spans smaller wave numbers down to the characteristic $k_{\mathrm{IR}}=2 \pi / L$ of each lattice.

In summary, the expected GW plateau always emerges during RD in all our simulations with different $N$ and $d x$. The length of the plateau varies however depending on the IR coverage of each simulation, and in the smaller-volume simulations it is not readily apparent.

\section{SUMMARY AND DISCUSSION}

Cosmic defects are a natural by-product of a phase transition in the early Universe. The tensor metric perturbations they create are potentially observable as gravitational waves. The same tensor metric perturbations are also partly responsible for the B-mode polarization signal created by cosmic defects in the CMB.

In this paper, we have calculated the GW spectra for defects from $O(N)$ global symmetry breaking in two different ways: integrating the anisotropic stress unequaltime correlator with the Green's functions for the tensor metric perturbations, and performing real-time simulations of the tensor perturbations sourced by the evolving defects. We found good agreement, numerically demonstrating the equivalence of the two methods, and providing a robust check on the results.

Our results are consistent with, and improve upon, those in Paper I [117]. The improvement is in two ways: we have extended the frequency range down to the Hubble rate today and beyond, giving a formal way to extend the power spectrum to super-Hubble scales. The numerical simulations are also twice as large, reducing uncertainties on the GW spectrum.

The question arises then of whether we could directly detect those tensor modes with GW experiments. Given the smallness of the frequency today corresponding to the horizon scale at the time of matter-radiation equality, cf. Eq. (43), direct GW detection experiments can only potentially probe the $\mathrm{GW}$ background produced during $\mathrm{RD}$, corresponding to frequencies $f \gg f_{\text {eq }} \simeq 6.6 \times 10^{-17} \mathrm{~Hz}$. Therefore, in order to assess the potential observability of the GW background from defects, only the GW plateau amplitude is relevant. Using Eqs. (45) and (46), together with Eq. (58), we obtain that the GW plateau amplitude today is

$$
\begin{aligned}
h_{0}^{2} \Omega_{\mathrm{GW}}^{(0)} & \simeq h_{0}^{2} \Omega_{\mathrm{rad}}^{(0)} \times \Sigma_{N} \times \frac{650}{N}\left(\frac{v}{M_{\mathrm{Pl}}}\right)^{4} \\
& \simeq 2.63 \times 10^{-15} \times \frac{\Sigma_{N}}{N} \times\left(\frac{G \mu}{10^{-6}}\right)^{2},
\end{aligned}
$$

where we have used $h_{0}^{2} \Omega_{\mathrm{rad}}^{(0)} \simeq 4 \times 10^{-5}$, and we have introduced the dimensionless parameter

$$
G \mu \equiv \pi\left(\frac{v}{M_{\mathrm{Pl}}}\right)^{2} .
$$

As the largest plateau amplitudes are obtained for the lowest values of $N$, we can focus only on the case of global cosmic strings $(N=2)$ and monopoles $(N=3)$. In each case $\mu$ has a different meaning, e.g., for global strings it is the tension of the core of the string, whereas for global monopoles $\mu \delta$ it is roughly the energy stored in the monopole core, where $\delta$ is the width of the monopole.

Based on the latest Planck results, CMB constraints on such global strings and monopoles, lead to [87]

$$
\begin{array}{ll}
10^{12}(G \mu)^{2}<0.031, & N=2 \\
10^{12}(G \mu)^{2}<0.73, & N=3 .
\end{array}
$$

In this work, we have quantified the ratio between the numerical and large- $N$ analytical GW spectra, as

$$
\frac{\Sigma_{N}}{N} \simeq \begin{cases}119, & N=2, \\ 3.33, & N=3,\end{cases}
$$

as shown in Table I. Using Eqs. (77) and (78), the maximum amplitude of the GW plateau (75) that we can obtain, is then 


$$
\begin{array}{ll}
h_{0}^{2} \Omega_{\mathrm{GW}}^{(0)}<9.7 \times 10^{-15}, & N=2, \\
h_{0}^{2} \Omega_{\mathrm{GW}}^{(0)}<6.4 \times 10^{-15}, & N=3 .
\end{array}
$$

These amplitudes are larger than the maximum amplitude expected (as bounded by current CMB constraints $[144,145])$ for the quasi-scale-invariant GW background in slow-roll inflation [20], $h_{0}^{2} \Omega_{\mathrm{GW}}^{\text {(inf) }} \lesssim 10^{-16}$. The amplitudes in Eq. (79) are however too small to be observed by any planned direct GW detection experiment. For instance, based on the projected capabilities of LISA to detect a stochastic GW background [21], we conclude that the GW plateau for global strings cannot be detected by LISA with any significant signal-to-noise ratio, as this would require at least $h_{0}^{2} \Omega_{\mathrm{GW}}^{(0)} \gtrsim 10^{-13}$ at the LISA sensitivity peak of $f_{p} \sim 10^{-3} \mathrm{~Hz}$. The proposed BBO satellite mission would be able to improve on the CMB limits, having a projected sensitivity to a cosmic background of $h_{0}^{2} \Omega_{\mathrm{GW}}^{(0)} \gtrsim 10^{-17}$ [146]. DECIGO [15,16,147-149], with similar expected sensitivity, could possibly also detect the signal coming from global strings.

We note that the factor $\sim 2$ of discrepancy between our present $N=2$ result and Paper I, might be a signal of the difficulty in establishing the scaling properties of a global string network. Indeed logarithmic departures from standard scaling $\xi \propto \tau$ have been reported [150]. However, in a dedicated study about the scaling density of axion strings [121], some of us found that such a logarithmic correction to the long-term scaling is not clearly supported by the data, and is better understood as a feature of the approach to scaling from low string densities. In any case, the bound on the GWs from strings we report here should be understood as valid only under the assumption of standard scaling.

The case $N=2$ is of special physical interest, because global strings are an inevitable consequence of global $U(1)$ symmetry breaking after inflation, and are therefore associated with axion-like particle (ALP) dark matter models. In recent years there has been a revived interest in studying such axion strings; see e.g., Refs. [123,150-156]. A GW signal from axion strings could be complementary to current detection strategies for axion-like particles. In the case of hidden axion sectors with no interaction with the SM (other than gravitational), it might represent, potentially, the only accessible signal. Given the relevance of this case, and the need to control the technical difficulties commented above, we plan to study the case of global strings in more detail elsewhere.

Let us now compare our results to other studies of GWs from global defects. Reference [157] presented simulations of the real-time evolution of tensors, similar to our simulations in Sec. V, but introduced an early MD epoch (due to quadratic inflaton oscillations during reheating) before the onset of RD. The numerical GW energy density spectrum they obtained exhibits the expected high frequency tail $\Omega_{\mathrm{GW}} \propto 1 / f^{2}$ for the modes that crossed the horizon during $\mathrm{MD}$, as expected. The GW spectra also exhibit a bump at the IR/intermediate scales that cross the horizon during RD. As the background dynamics goes deeper and deeper into RD, the bump grows and seems to flatten; a tail $\Omega_{\mathrm{GW}} \propto f^{3}$ is however always visible at the most IR scales captured in their simulations; see for instance the low-frequency part of the GW spectra in Fig. 2 of Ref. [157]. Although there is no clear plateau, the authors interpreted their peak power as an estimate of its value, while expressing caution that a greater dynamical range than that afforded by their $N_{p}=512$ points/dimension is needed. Indeed, we found that $N_{p}=2048$ points/ dimension and a large lattice spacing $(d \tilde{x}=1)$ were needed to show a clear plateau developed in the IR, and even then it spanned no more than roughly one decade of scales (Figs. 5 and 8).

More recently, Ref. [158] studied the GW emission from oscillating loops chopped off from the network of global strings, using a Nambu-Goto approximation to their dynamics (see also Ref. [159]). Using the velocitydependent one-scale model for the string network evolution [160-162], the authors concluded that the emission of GWs by oscillating loops can be significantly greater than the GW signal we obtained in the present work, estimating $h_{0}^{2} \Omega_{\mathrm{GW}}^{(0)} \sim 10^{-12}$ at LISA frequencies for $G \mu \sim 10^{-7}$, whereas we would predict $h_{0}^{2} \Omega_{\mathrm{GW}}^{(0)} \sim 10^{-14}$.

Their results are based on analytical studies that expect the loops to sustain both GW and Goldstone emissions [81,163]: if the Goldstone decay channel is slow enough, GWs will be emitted by each loop for as long as they exist, and hence a significant stochastic background of GWs will build up from the contribution of all loops during their lifetime. While we have not performed a detailed analysis of the loops in our simulations, it is known that loops of Abelian Higgs strings produced in field theory network simulations decay much more rapidly than predicted in the Nambu-Goto approach [164], and this is very likely also to be true for global strings. Indeed, a recent dedicated lattice study of the decay of global string loops [165], concluded that the global string loop lifetime is of the order of the initial loop length $L$. This is in concordance with our numerical results, in the sense that in our GW computation we included the contribution from every possible field configuration in the string network, including that of loops, and we did not observe an accumulated emission of GW radiation from long-lived loops. These aspects will require further investigation, particularly given the relevance of the case of global strings in relation to ALP dark matter models.

\section{ACKNOWLEDGMENTS}

D. G. F. is supported by a Ramón y Cajal contract by Spanish Ministry Ministerio de Economía, Industria y Competitividad (MINECO), with Ref. RYC-2017-23493. 
D. G. F. acknowledges hospitality and support from Kavli Institute for Theoretical Physics (KITP) in Santa Barbara, where part of this work was completed. M. H. acknowledges support from the Science and Technology Facilities Council (Grant No. ST/L000504/1). J. L. and J. U. acknowledge support from Eusko Jaurlaritza (IT-979-16) and Ministerio de Ciencia, Innovación y Universidades / Agencia Estatal de Investigación / Fondo Europeo de Desarrollo Regional (MCIU/AEI/FEDER) grant Fondo Europeo de Desarrollo Regional (Grant No. PGC2018094626-B-C21). This work has been possible thanks to the computing infrastructure of the ARINA cluster at the University of the Basque Country, UPV/EHU. This research was also supported in part by the National Science Foundation under Grant No. NSF PHY-1748958.

\section{APPENDIX A: ANALYTIC SOLUTION IN THE LARGE- $N$ LIMIT}

Let us consider a global theory where an $O(N)$ symmetry is spontaneously broken into $O(N-1)$. The starting point is an $N$-component scalar field $\Phi=\left(\phi_{1}, \phi_{2}, \ldots, \phi_{N}\right)^{\mathrm{T}} / \sqrt{2}$ with the Lagrangian given by Eq. (44). For $N \gg 1$, the dynamics of the Goldstone modes can be well described by a nonlinear sigma model $[115,135]$, where we force the vacuum constraint $\sum_{a} \phi_{a}^{2}(\mathbf{x}, t)=v^{2}$ by a Lagrange multiplier, where $v$ is the VEV. Normalizing the symmetrybreaking field to its VEV, $\beta^{a} \equiv \phi^{a} / v$, each component of the field obeys the nonlinear sigma model evolution equation

$$
\square \beta^{a}-\left(\partial_{\mu} \beta \cdot \partial^{\mu} \beta\right) \beta^{a}=0,
$$

where $\quad\left(\partial_{\mu} \beta \cdot \partial^{\mu} \beta\right)=\sum_{a} \eta^{\mu \nu} \partial_{\mu} \beta^{a}(\mathbf{x}, t) \partial_{\nu} \beta^{a}(\mathbf{x}, t) \quad$ and $|\beta(\mathbf{x}, t)|^{2} \equiv \sum_{a} \beta^{a}(\mathbf{x}, t) \beta^{a}(\mathbf{x}, t)=1$. In the large- $N$ limit, we can replace the sum over components by an ensemble average $T(x)=\sum_{a} \eta^{\mu \nu} \partial_{\mu} \beta^{a} \partial_{\nu} \beta^{a}=N\left\langle\eta^{\mu \nu} \partial_{\mu} \beta^{a} \partial_{\nu} \beta^{a}\right\rangle=\bar{T}(t)$. By dimensional considerations, $T \propto \mathcal{H}^{2}$, or $\bar{T}(t)=T_{0} t^{-2}$, with $T_{0}>0$. Replacing the nonlinearity in Eq. (A1) by this expectation value, and Fourier transforming, we obtain a linear equation

$$
\ddot{\beta}_{k}^{a}+\frac{2 \gamma}{t} \dot{\beta}_{k}^{a^{\prime}}+\left(k^{2}-\frac{T_{0}}{t^{2}}\right) \beta_{k}^{a}=0,
$$

where $\gamma \equiv d \log a / d \log \eta$, with e.g., $\gamma=1$ for RD or $\gamma=2$ for MD. The solution to Eq. (A2) for constant $\gamma$, and preserving the vacuum manifold constraint $|\beta(\mathbf{x}, t)|^{2}=1$, is given by $T_{0}=3(\gamma+1 / 4)$ and

$$
\beta^{a}(\mathbf{k}, t)=\sqrt{A}\left(\frac{t}{t_{*}}\right)^{\frac{3}{2}} \frac{J_{\nu}(k t)}{(k t)^{\nu}} \beta^{a}\left(\mathbf{k}, t_{*}\right),
$$

where $J_{\nu}(x)$ are Bessel functions of order $\nu \equiv \gamma+1$, and $A \equiv 4 \Gamma(2 \nu-1 / 2) \Gamma(\nu-1 / 2) / 3 \Gamma(\nu-1)$.
Here $\beta^{a}\left(k, t_{*}\right)$ is the $a$ th component of the field at the initial time $t_{*}$, and the normalization constant $A$ has been determined by imposing the condition $\left\langle\left|\beta\left(\mathbf{x}, t_{*}\right)\right|^{2}\right\rangle=1$ at the initial time. The analytical solution (A3) shows explicitly that the self-ordering dynamics of the nontopological defects exhibits scaling. The condition $\beta^{2}=1$ actually introduces correlations between the different components of $\beta$, but these lead to corrections of order $1 / N$ [166], which in the large- $N$ limit can be neglected. On large scales, $\beta^{2}(\mathbf{x}, t) \simeq\left\langle\beta^{2}(\mathbf{x}, t)\right\rangle(1+\mathcal{O}(1 / N))$ is a very good approximation at all times $t \geq t_{*}$. See Ref. [120] for details.

In the limit $N \gg 1$, it is also possible to analytically calculate the GW power spectrum emitted by the evolution of the resulting self-ordering process of the nontopological textures. The GW amplitude for modes entering the horizon during RD was calculated in Ref. [120] as follows. Starting from Eq. (22) we just need to calculate $\Pi^{2}\left(k, t, t^{\prime}\right)$ [see Eq. (19)] using the solution Eq. (A3). As we only care about the field gradients as a source of GWs, the TT part of the effective anisotropic stress tensor is $\Pi_{i j}^{\mathrm{TT}}=\left\{\partial_{\mu} \phi^{a} \partial_{\nu} \phi^{a}\right\}^{\mathrm{TT}}$, which in Fourier space reads

$\Pi_{i j}^{\mathrm{TT}}(\mathbf{k}, t)=v^{2} \int \frac{d^{3} q}{(2 \pi)^{3}} q_{l} \Lambda_{i j, l m}(\hat{\mathbf{k}}) q_{m} \beta^{a}(\mathbf{q}, t) \beta^{a}(\mathbf{k}-\mathbf{q}, t)$.

The UETC defined by Eq. (19) can then be written as

$$
\begin{aligned}
\left\langle\Pi_{i j}^{\mathrm{TT}}(\mathbf{k}, t) \Pi_{i j}^{\mathrm{TT}}\left(\mathbf{k}^{\prime}, t^{\prime}\right)\right\rangle & \\
= & v^{4} \int \frac{d^{3} q d^{3} q^{\prime}}{(2 \pi)^{6}}\left(q^{\mathrm{T}} \Lambda q\right)_{i j}\left(q^{\prime \mathrm{T}} \Lambda q^{\prime}\right)_{l m} \\
& \times\left\langle\beta^{a}(\mathbf{q}, t) \beta^{a}(\mathbf{k}-\mathbf{q}, t) \beta^{* b}\left(\mathbf{q}^{\prime}, t^{\prime}\right) \beta^{* b}\left(\mathbf{k}-\mathbf{q}, t^{\prime}\right)\right\rangle \\
\equiv & (2 \pi)^{3} \Pi^{2}\left(k, t, t^{\prime}\right) \delta^{(3)}\left(\mathbf{k}-\mathbf{k}^{\prime}\right),
\end{aligned}
$$

where we used the notation $\left(q^{\mathrm{T}} \Lambda q\right)_{i j} \equiv q_{l} \Lambda_{i j, l m} q_{m}$.

It can be shown [166] that in the large- $N$ limit the field $\beta$ is Gaussian distributed initially, up to corrections $\sim 1 / N$. As its time evolution is linear, $\beta$ will remain a Gaussian field, so we can determine higher-order correlators via Wick's theorem. This is relevant in order to compute the UETC given in Eq. (A5), characterized by the four-point field correlator. By means of Wick's theorem, we can reduce the four-point function of the self-ordering fields to products of two-point functions, $\langle\beta \beta \beta \beta\rangle \sim \sum_{\text {pairs }}\langle\beta \beta\rangle$. One obtains $[120,167]$

$$
\begin{aligned}
\Pi^{2}\left(k, t, t^{\prime}\right)= & v^{4} \int \frac{d^{3} q}{(2 \pi)^{3}} q^{4}\left[1-(\hat{\mathbf{k}} \cdot \hat{\mathbf{q}})^{2}\right]^{2} \\
& \times \mathcal{P}_{\beta}^{a b}\left(|\mathbf{q}|, t, t^{\prime}\right) \mathcal{P}_{\beta}^{a b}\left(|\mathbf{k}-\mathbf{q}|, t, t^{\prime}\right),
\end{aligned}
$$

where

$\left\langle\beta^{a}(\mathbf{k}, \eta) \beta^{* b}\left(\mathbf{k}^{\prime}, t^{\prime}\right)\right\rangle \equiv(2 \pi)^{3} \mathcal{P}_{\beta}^{a b}\left(k, t, t^{\prime}\right) \delta\left(\mathbf{k}-\mathbf{k}^{\prime}\right)$. 
If we assume that $\beta$ is initially aligned on scales smaller than the comoving horizon $t_{*}$, and that it has an arbitrary orientation on scales larger than $t_{*}$, this corresponds to a white noise spectrum on large scales and vanishing power on small scales,

$$
\left\langle\beta^{a}\left(\mathbf{k}, t_{*}\right) \beta^{* b}\left(\mathbf{k}^{\prime}, t_{*}\right)\right\rangle=(2 \pi)^{3} 6 \pi^{2} t_{*}^{3} \frac{\delta^{a b}}{N} \Theta\left(1-k t_{*}\right) \delta\left(\mathbf{k}-\mathbf{k}^{\prime}\right)
$$

where we have neglected the details of the decay of the correlator around $k \eta_{*}=1$, and simply modeled it with a Heaviside function. The amplitude $6 \pi^{2} t_{*}^{3}$ at superhorizon scales is determined by imposing the condition $\beta^{2}\left(\mathbf{x}, \eta_{*}\right) \simeq$ $\left\langle\beta^{2}\left(\mathbf{x}, \eta_{*}\right)\right\rangle$ everywhere in space (up to corrections of order $1 / N)$. Using Eqs. (A3) and (A8), we finally arrive at

$$
\begin{aligned}
\mathcal{P}_{\beta}^{a b}\left(k, t, t^{\prime}\right) & =\frac{\delta_{a b}}{N} 6 \pi^{2} A\left(t t^{\prime}\right)^{3 / 2} \frac{J_{\nu}(k t) J_{\nu}\left(k t^{\prime}\right)}{(k t)^{\nu}\left(k t^{\prime}\right)^{\nu}} \\
& \equiv \frac{\delta_{a b}}{N} f(k, t) f\left(k, t^{\prime}\right),
\end{aligned}
$$

with $f(k, t) \equiv \pi \sqrt{6 A} k^{3 / 2} J_{\nu}(k t)(k t)^{3 / 2-\nu}$. Note that this corresponds to a totally coherent source, in the sense that its unequal-time correlator $\mathcal{P}_{\beta}^{a b}\left(k, t, t^{\prime}\right)$ is a product of a function of $t$ and $t^{\prime}$.

Combining Eqs. (22), (A6), and (A9), we arrive at

$$
\begin{aligned}
\frac{d \rho_{\mathrm{GW}}(k, t)}{d \log k}= & \frac{G v^{4}}{4 \pi^{4}} \frac{k^{3}}{a^{4}(t)} \int d t^{\prime} d t^{\prime \prime} a\left(t^{\prime}\right) a\left(t^{\prime \prime}\right) \int d \mathbf{p}|\mathbf{p}|^{4} \\
& \times \sin ^{4} \theta \cos \left(k\left(t^{\prime}-t^{\prime \prime}\right)\right) \mathcal{P}_{\beta}^{a b}\left(p, t^{\prime}, t^{\prime \prime}\right) \\
& \times \mathcal{P}_{\beta}^{a b}\left(|\mathbf{k}-\mathbf{p}|, t^{\prime}, t^{\prime \prime}\right) .
\end{aligned}
$$

This formula is actually valid for describing the energy density spectra during either RD or MD, by simply choosing the appropriate scale factor behavior and value of $\nu$ in Eq. (A9) ( $\nu=2$ for RD and $\nu=3$ for MD). For instance, by numerically integrating Eq. (A10) with $\nu=2$ and $a(t) \simeq \sqrt{\Omega_{\mathrm{rad}}^{(0)}} a_{0}^{2} H_{0} t$, one obtains the spectral plateau amplitude for the modes emitted during RD [120,122], that we reported in Eq. (46).

\section{APPENDIX B: LATTICE FORMULATION OF THE SCALAR FIELD EQUATIONS}

The model under study is a model with an $N$-component scalar field $\Phi=\left(\phi_{1}, \phi_{2}, \ldots, \phi_{N}\right)^{\mathrm{T}} / \sqrt{2}$, with the Lagrangian given in Eq (44). This Lagrangian has an $O(N)$ symmetry that is spontaneously broken to $O(N-1)$. From this Lagrangian, one can obtain the corresponding EOMs, which are the ones given in Eq. (47).

In order to simulate the model in a lattice, we need the discrete version of those EOMs. The approach we follow consists in the discretization of the action [128], and from it we derive the EOMs, rather than discretizing the EOMs directly. Then, we use a leapfrog method to solve the equations on the lattice.

Before discretization, we introduce the following dimensionless variables: $\phi_{a} \rightarrow \phi_{a} / v, d x \rightarrow m_{\mathrm{c}} d x$, with $m_{\mathrm{c}} \equiv$ $\sqrt{\lambda_{c}} v$ and $\lambda_{c}=a^{2} \lambda=$ const. With these changes, the discretized action reads

$$
\begin{aligned}
S_{\mathrm{lat}}= & \Delta t \Delta x^{3} \sum_{t, x}\left[\frac{1}{2} a(t+\delta t) \sum_{a=1}^{N} \pi_{a}(\vec{x}, t+\delta t)^{2}\right. \\
& -\frac{1}{2} a(t)^{2} \sum_{a=1}^{N} \sum_{i=1}^{3}\left(\frac{\phi_{a}\left(\vec{x}+\delta \vec{x}_{i}, t\right)-\phi_{a}(\vec{x}, t)}{\Delta x}\right) \\
& \left.-\frac{1}{4} a(t)^{4}\left(\sum_{a=1}^{N}\left(\phi_{a}(\vec{x}, t)\right)^{2}-1\right)^{2}\right],
\end{aligned}
$$

where $\delta \vec{x}_{i}$ denotes the three directions on the lattice, e.g., $\phi_{a}\left(\vec{x}+\delta \vec{x}_{2}, t\right)=\phi_{a}(x, y+\Delta x, z, t), \delta t=\Delta t / 2$, and we have defined the lattice conjugate momenta of the fields as

$$
\pi_{a}(\vec{x}, t+\delta t)=\frac{1}{\Delta t}\left(\phi_{a}(\vec{x}, t+\Delta t)-\phi_{a}(\vec{x}, t)\right) .
$$

Note that while the field $\Phi$ lives in integer time steps, the conjugate momentum $\Pi=\left(\pi_{1}, \pi_{2}, \ldots, \pi_{N}\right)$ lives in halfinteger time steps, as needed for the leapfrog method, and the times where the scale factor $a$ is evaluated are chosen accordingly.

We can now obtain the discretized EOMs from this discretized action

$$
\begin{aligned}
\pi_{a}(\vec{x}, t+\delta t)= & \left(\frac{a(t-\delta t)}{a(t+\delta t)}\right)^{2} \pi_{a}(\vec{x}, t-\delta t)+\Delta t\left[\left(\frac{a(t)}{a(t+\delta t)}\right)^{2} \sum_{i=1}^{3} \frac{\phi_{a}\left(\vec{x}+\delta \vec{x}_{i}, t\right)+\phi_{a}\left(\vec{x}-\delta \vec{x}_{i}, t\right)-2 \phi_{a}(\vec{x}, t)}{\Delta x^{2}}\right. \\
& \left.-\left(\frac{a(t)^{2}}{a(t+\delta t)}\right)^{2}\left(\sum_{b=1}^{N}\left(\phi_{b}(\vec{x}, t)\right)^{2}-1\right) \phi_{a}(\vec{x}, t)\right]
\end{aligned}
$$

which together with the equation for $\phi_{a}(\vec{x}, t+\Delta t)$ obtained from Eq. (B2)

$$
\phi_{a}(\vec{x}, t+\Delta t)=\phi_{a}(\vec{x}, t)+\Delta t \pi_{a}(\vec{x}, t+\delta t)
$$


are the equations used to update the values of the fields as the dynamics progresses.

\section{APPENDIX C: GWs ON THE LATTICE}

We now discuss the discretization to obtain the spectrum of GWs on the lattice, following Ref. [168]. We have seen in the main text that the spectrum of the energy density of a (statistically) homogeneous and isotropic GW background in the continuum is given by Eq. (12), which we reproduce here for ease of reading:

$$
\frac{d \rho_{\mathrm{GW}}}{d \log k}=\frac{k^{3}}{(4 \pi)^{3} G a^{2}(t) V} \int \frac{d \Omega_{k}}{4 \pi} \dot{h}_{i j}(\mathbf{k}, t) \dot{h}_{i j}^{*}(\mathbf{k}, t)
$$

where $d \Omega_{k}$ represents a solid angle element in $\mathbf{k}$ space. The previous equation is valid in the limit of a very large volume $V$ encompassing all relevant wavelengths. The same condition on the lattice means that the volume $V=L^{3}=$ $(N d x)^{3}$ needs to encompass sufficiently well the characteristic wavelengths of the simulated GW background.

In order to derive an analogous discrete expression to Eq. (C1) for a lattice of volume $V=N^{3} d x^{3}$, we first need to specify our discrete Fourier transform (DFT) convention. We use

$$
\begin{aligned}
& f(\mathbf{n})=\frac{1}{N^{3}} \sum_{\tilde{n}} e^{-\frac{2 \pi i}{N} \tilde{\mathbf{n}}} \tilde{f}(\tilde{\mathbf{n}}), \\
& \tilde{f}(\tilde{\mathbf{n}})=\sum_{n} e^{+\frac{2 \pi i}{N} \tilde{\mathbf{n}}} f(\mathbf{n}),
\end{aligned}
$$

where the index $\mathbf{n}=\left(n_{1}, n_{2}, n_{3}\right)$, with $n_{i}=0,1, \ldots, N-1$, labels our lattice sites in configuration space, whereas the index $\tilde{\mathbf{n}}=\left(\tilde{n}_{1}, \tilde{n}_{2}, \tilde{n}_{3}\right)$ labels the reciprocal lattice, with $\tilde{n}_{i}=-\frac{N}{2}+1,-\frac{N}{2}+2, \ldots-1,0,1, \ldots, \frac{N}{2}$. Following Ref. [168], one arrives at

$$
\begin{aligned}
\left(\frac{d \rho_{G W}}{d \log k}\right)(\tilde{\mathbf{n}}) \equiv & \frac{|k(\tilde{\mathbf{n}})|^{3}}{(4 \pi)^{3} G a^{2}(t) L^{3}} \cdot \\
& \cdot\left\langle\left[d x^{3} \dot{h}_{i j}(|\tilde{\mathbf{n}}|, t)\right]\left[d x^{3} \dot{h}_{i j}(|\tilde{\mathbf{n}}|, t)\right]^{*}\right\rangle,
\end{aligned}
$$

where $\left\langle\dot{h}_{i j}(|\tilde{\mathbf{n}}|, t) \dot{h}_{i j}^{*}(|\tilde{\mathbf{n}}|, t)\right\rangle$ is an average over configurations with lattice momenta $\tilde{\mathbf{n}}^{\prime} \in[|\tilde{\mathbf{n}}|,|\tilde{\mathbf{n}}|+\delta \tilde{n}]$. The DFT becomes the continuous Fourier transform (CFT) in the continuum limit DFT $\left\{f(\mathbf{n}) d x^{3}\right\} \rightarrow \operatorname{CFT}\{f(\mathbf{x})\}$. Therefore, the expression $(\mathrm{C} 4)$ matches the expression $(\mathrm{C} 1)$ in the continuum limit. Equation (C4) highlights that the natural momenta in terms of which the lattice GW spectrum should be expressed are the discretized versions of the continuum ones $\mathbf{k}=\tilde{\mathbf{n}} k_{\mathrm{IR}}$, and not any of the lattice momenta that one can define based on the choice of a lattice derivative.

As mentioned in the main text, the TT metric perturbations follow Eq. (4) in the continuum, where the TT part of a tensor is more easily obtained in Fourier space by means of the projectors $\Lambda_{i j, l m}(\hat{\mathbf{k}})$ given in Eq. (6), which we also reproduce here:

$$
\begin{aligned}
\Lambda_{i j, l m}(\hat{\mathbf{k}}) & \equiv P_{i l}(\hat{\mathbf{k}}) P_{j m}(\hat{\mathbf{k}})-\frac{1}{2} P_{i j}(\hat{\mathbf{k}}) P_{l m}(\hat{\mathbf{k}}), \\
P_{i j} & =\delta_{i j}-\hat{k}_{i} \hat{k}_{j}, \quad \hat{k}_{i}=k_{i} / k .
\end{aligned}
$$

On the lattice, one can construct a different projector for each different discretization of the spatial derivative, and in Ref. [168] it was shown that those different projectors give rise to differences in the very UV part of the GW spectrum. Nevertheless, for our case, those differences, after integrating the spectrum over its Fourier modes, amount only to a few percent. Thus, all projectors are equivalent within those errors, and the projectors used in this work are the ones based on nearest-neighbor spatial derivatives [168]:

$$
\begin{aligned}
\Lambda_{i j, l m}^{(L)}(\tilde{\mathbf{n}}) & \equiv P_{i l}^{(L)}(\tilde{\mathbf{n}}) P_{j m}^{(L)}(\tilde{\mathbf{n}})-\frac{1}{2} P_{i j}^{(L)}(\tilde{\mathbf{n}}) P_{l m}^{(L)}(\tilde{\mathbf{n}}), \\
P_{i j}^{(L)}(\tilde{\mathbf{n}}) & =\delta_{i j}-\frac{k_{i}^{(L)} k_{j}^{(L)}}{\left|k^{(L)}\right|^{2}} \\
k_{i}^{(L)} & =2 \frac{\sin \left(\pi \tilde{n}_{i} / N\right)}{d x} .
\end{aligned}
$$

[1] B. P. Abbott et al. (LIGO Scientific and Virgo Collaborations), Phys. Rev. Lett. 116, 061102 (2016).

[2] B. P. Abbott et al. (LIGO Scientific and Virgo Collaborations), Phys. Rev. Lett. 116, 241103 (2016).

[3] B. P. Abbott et al. (LIGO Scientific and Virgo Collaborations), Phys. Rev. Lett. 118, 221101 (2017); 121, 129901 (E) (2018).

[4] B. P. Abbott et al. (LIGO Scientific and Virgo Collaborations), Astrophys. J. 851, L35 (2017).
[5] B. P. Abbott et al. (LIGO Scientific and Virgo Collaborations), Phys. Rev. Lett. 119, 141101 (2017).

[6] G. M. Harry (LIGO Scientific Collaboration), Classical Quantum Gravity 27, 084006 (2010).

[7] F. Acernese (Virgo Collaboration), J. Phys. Conf. Ser. 610, 012014 (2015).

[8] K. Somiya (KAGRA Collaboration), Classical Quantum Gravity 29, 124007 (2012).

[9] C. S. Unnikrishnan, Int. J. Mod. Phys. D 22, 1341010 (2013). 
[10] http://www.ligo-india.in/.

[11] B. Sathyaprakash et al., Classical Quantum Gravity 29, 124013 (2012); 30, 079501(E) (2013).

[12] P. Amaro-Seoane et al. (LISA Collaboration), arXiv: 1702.00786.

[13] W.-R. Hu and Y.-L. Wu, Natl. Sci. Rev. 4, 685 (2017).

[14] W.-H. Ruan, Z.-K. Guo, R.-G. Cai, and Y.-Z. Zhang, Int. J. Mod. Phys. A 35, 2050075 (2020).

[15] N. Seto, S. Kawamura, and T. Nakamura, Phys. Rev. Lett. 87, 221103 (2001).

[16] S. Kawamura et al., Classical Quantum Gravity 23, S125 (2006).

[17] J. Crowder and N. J. Cornish, Phys. Rev. D 72, 083005 (2005).

[18] B. P. Abbott et al. (LIGO Scientific and Virgo Collaborations), Phys. Rev. D 100, 061101 (2019).

[19] A. Renzini and C. Contaldi, Phys. Rev. D 100, 063527 (2019).

[20] C. Caprini and D. G. Figueroa, Classical Quantum Gravity 35, 163001 (2018).

[21] C. Caprini, D. G. Figueroa, R. Flauger, G. Nardini, M. Peloso, M. Pieroni, A. Ricciardone, and G. Tasinato, J. Cosmol. Astropart. Phys. 11 (2019) 017.

[22] L. P. Grishchuk, Zh. Eksp. Teor. Fiz. 67, 825 (1974) [Sov. Phys. JETP 40, 409 (1975)].

[23] A. A. Starobinsky, JETP Lett. 30, 682 (1979); 30, 767 (1979).

[24] V. A. Rubakov, M. V. Sazhin, and A. V. Veryaskin, Phys. Lett. 115B, 189 (1982).

[25] R. Fabbri and M. d. Pollock, Phys. Lett. 125B, 445 (1983).

[26] M. M. Anber and L. Sorbo, J. Cosmol. Astropart. Phys. 10 (2006) 018.

[27] L. Sorbo, J. Cosmol. Astropart. Phys. 06 (2011) 003.

[28] E. Pajer and M. Peloso, Classical Quantum Gravity 30, 214002 (2013).

[29] R. Namba, M. Peloso, M. Shiraishi, L. Sorbo, and C. Unal, J. Cosmol. Astropart. Phys. 01 (2016) 041.

[30] R. Z. Ferreira, J. Ganc, J. Noreña, and M. S. Sloth, J. Cosmol. Astropart. Phys. 04 (2016) 039; 10 (2016) E01.

[31] M. Peloso, L. Sorbo, and C. Unal, J. Cosmol. Astropart. Phys. 09 (2016) 001.

[32] V. Domcke, M. Pieroni, and P. Binétruy, J. Cosmol. Astropart. Phys. 06 (2016) 031.

[33] N. Bartolo et al., J. Cosmol. Astropart. Phys. 12 (2016) 026.

[34] B. Thorne, T. Fujita, M. Hazumi, N. Katayama, E. Komatsu, and M. Shiraishi, Phys. Rev. D 97, 043506 (2018).

[35] M. Giovannini, Phys. Rev. D 58, 083504 (1998).

[36] M. Giovannini, Phys. Rev. D 60, 123511 (1999).

[37] L. A. Boyle and A. Buonanno, Phys. Rev. D 78, 043531 (2008).

[38] D. G. Figueroa and E. H. Tanin, J. Cosmol. Astropart. Phys. 10 (2019) 050.

[39] D. G. Figueroa and E. H. Tanin, J. Cosmol. Astropart. Phys. 08 (2019) 011.

[40] N. Bernal and F. Hajkarim, Phys. Rev. D 100, 063502 (2019).

[41] R. Easther and E. A. Lim, J. Cosmol. Astropart. Phys. 04 (2006) 010.
[42] J. Garcia-Bellido and D. G. Figueroa, Phys. Rev. Lett. 98, 061302 (2007).

[43] J. Garcia-Bellido, D. G. Figueroa, and A. Sastre, Phys. Rev. D 77, 043517 (2008).

[44] J. F. Dufaux, A. Bergman, G. N. Felder, L. Kofman, and J.-P. Uzan, Phys. Rev. D 76, 123517 (2007).

[45] J.-F. Dufaux, G. Felder, L. Kofman, and O. Navros, J. Cosmol. Astropart. Phys. 03 (2009) 001.

[46] J.-F. Dufaux, D. G. Figueroa, and J. Garcia-Bellido, Phys. Rev. D 82, 083518 (2010).

[47] K. Enqvist, D. G. Figueroa, and T. Meriniemi, Phys. Rev. D 86, 061301 (2012).

[48] D. G. Figueroa and T. Meriniemi, J. High Energy Phys. 10 (2013) 101.

[49] D. G. Figueroa, J. High Energy Phys. 11 (2014) 145.

[50] D. G. Figueroa, J. García-Bellido, and F. Torrentí, Phys. Rev. D 93, 103521 (2016).

[51] D. G. Figueroa and F. Torrenti, J. Cosmol. Astropart. Phys. 10 (2017) 057.

[52] P. Adshead, J. T. Giblin, and Z. J. Weiner, Phys. Rev. D 98, 043525 (2018).

[53] P. Adshead, J. T. Giblin, M. Pieroni, and Z. J. Weiner, Phys. Rev. Lett. 124, 171301 (2020).

[54] P. Adshead, J. T. Giblin, M. Pieroni, and Z. J. Weiner, Phys. Rev. D 101, 083534 (2020).

[55] S.-Y. Zhou, E. J. Copeland, R. Easther, H. Finkel, Z.-G. Mou, and P. M. Saffin, J. High Energy Phys. 10 (2013) 026.

[56] S. Antusch, F. Cefala, and S. Orani, Phys. Rev. Lett. 118, 011303 (2017); 120, 219901(E) (2018).

[57] S. Antusch, F. Cefala, and S. Orani, J. Cosmol. Astropart. Phys. 03 (2018) 032.

[58] J. Liu, Z.-K. Guo, R.-G. Cai, and G. Shiu, Phys. Rev. Lett. 120, 031301 (2018).

[59] M. A. Amin, J. Braden, E. J. Copeland, J. T. Giblin, C. Solorio, Z. J. Weiner, and S.-Y. Zhou, Phys. Rev. D 98, 024040 (2018).

[60] K. D. Lozanov and M. A. Amin, Phys. Rev. D 99, 123504 (2019).

[61] A. Kosowsky, M. S. Turner, and R. Watkins, Phys. Rev. Lett. 69, 2026 (1992).

[62] M. Kamionkowski, A. Kosowsky, and M. S. Turner, Phys. Rev. D 49, 2837 (1994).

[63] C. Caprini, R. Durrer, and G. Servant, Phys. Rev. D 77, 124015 (2008).

[64] S. J. Huber and T. Konstandin, J. Cosmol. Astropart. Phys. 09 (2008) 022.

[65] C. Caprini, R. Durrer, T. Konstandin, and G. Servant, Phys. Rev. D 79, 083519 (2009).

[66] C. Caprini, R. Durrer, and G. Servant, J. Cosmol. Astropart. Phys. 12 (2009) 024.

[67] M. Hindmarsh, S. J. Huber, K. Rummukainen, and D. J. Weir, Phys. Rev. Lett. 112, 041301 (2014).

[68] M. Hindmarsh, S. J. Huber, K. Rummukainen, and D. J. Weir, Phys. Rev. D 92, 123009 (2015).

[69] C. Caprini et al., J. Cosmol. Astropart. Phys. 04 (2016) 001.

[70] M. Hindmarsh, S. J. Huber, K. Rummukainen, and D. J. Weir, Phys. Rev. D 96, 103520 (2017). 
[71] D. Cutting, M. Hindmarsh, and D. J. Weir, Phys. Rev. D 97, 123513 (2018).

[72] R. Jinno, T. Konstandin, and M. Takimoto, J. Cosmol. Astropart. Phys. 09 (2019) 035.

[73] T. Vachaspati and A. Vilenkin, Phys. Rev. D 31, 3052 (1985).

[74] T. Damour and A. Vilenkin, Phys. Rev. Lett. 85, 3761 (2000).

[75] T. Damour and A. Vilenkin, Phys. Rev. D 64, 064008 (2001).

[76] T. Damour and A. Vilenkin, Phys. Rev. D 71, 063510 (2005).

[77] S. A. Sanidas, R. A. Battye, and B. W. Stappers, Phys. Rev. D 85, 122003 (2012).

[78] L. Sousa and P. P. Avelino, Phys. Rev. D 88, 023516 (2013).

[79] J. J. Blanco-Pillado and K. D. Olum, Phys. Rev. D 96, 104046 (2017).

[80] P. Auclair et al., J. Cosmol. Astropart. Phys. 04 (2020) 034.

[81] A. Vilenkin and E. P. S. Shellard, Cosmic Strings and Other Topological Defects (Cambridge University Press, Cambridge, England, 2000).

[82] M. B. Hindmarsh and T. W. B. Kibble, Rep. Prog. Phys. 58, 477 (1995).

[83] P. A. R. Ade et al. (Planck Collaboration), Astron. Astrophys. 571, A25 (2014).

[84] J. Lizarraga, J. Urrestilla, D. Daverio, M. Hindmarsh, M. Kunz, and A. R. Liddle, Phys. Rev. D 90, 103504 (2014).

[85] T. Charnock, A. Avgoustidis, E. J. Copeland, and A. Moss, Phys. Rev. D 93, 123503 (2016).

[86] J. Lizarraga, J. Urrestilla, D. Daverio, M. Hindmarsh, and M. Kunz, J. Cosmol. Astropart. Phys. 10 (2016) 042.

[87] A. Lopez-Eiguren, J. Lizarraga, M. Hindmarsh, and J. Urrestilla, J. Cosmol. Astropart. Phys. 07 (2017) 026.

[88] D. G. Figueroa, R. R. Caldwell, and M. Kamionkowski, Phys. Rev. D 81, 123504 (2010).

[89] C. Ringeval, Adv. Astron. 2010, 380507 (2010).

[90] D. Regan and M. Hindmarsh, J. Cosmol. Astropart. Phys. 03 (2015) 008.

[91] M. A. Amin and D. Grin, Phys. Rev. D 90, 083529 (2014).

[92] A. Vilenkin, Astrophys. J. 282, L51 (1984).

[93] J. K. Bloomfield and D. F. Chernoff, Phys. Rev. D 89, 124003 (2014).

[94] R. H. Brandenberger, Nucl. Phys. B293, 812 (1987).

[95] M. Srednicki and S. Theisen, Phys. Lett. B 189, 397 (1987).

[96] P. Bhattacharjee, C. T. Hill, and D. N. Schramm, Phys. Rev. Lett. 69, 567 (1992); 69, 520 (1991).

[97] T. Damour and A. Vilenkin, Phys. Rev. Lett. 78, 2288 (1997).

[98] U. F. Wichoski, J. H. MacGibbon, and R. H. Brandenberger, Phys. Rev. D 65, 063005 (2002).

[99] M. Peloso and L. Sorbo, Nucl. Phys. B649, 88 (2003).

[100] E. Sabancilar, Phys. Rev. D 81, 123502 (2010).

[101] T. Vachaspati, Phys. Rev. D 81, 043531 (2010).

[102] A. J. Long, J. M. Hyde, and T. Vachaspati, J. Cosmol. Astropart. Phys. 09 (2014) 030.

[103] P. Auclair, D. A. Steer, and T. Vachaspati, arXiv:1911 .12066 .

[104] A. Vilenkin, Phys. Lett. 107B, 47 (1981).
[105] F. S. Accetta and L. M. Krauss, Nucl. Phys. B319, 747 (1989).

[106] R. R. Caldwell and B. Allen, Phys. Rev. D 45, 3447 (1992).

[107] X. Siemens, V. Mandic, and J. Creighton, Phys. Rev. Lett. 98, 111101 (2007).

[108] M. R. DePies and C. J. Hogan, Phys. Rev. D 75, 125006 (2007).

[109] S. Olmez, V. Mandic, and X. Siemens, Phys. Rev. D 81, 104028 (2010).

[110] L. Sousa and P. P. Avelino, Phys. Rev. D 89, 083503 (2014).

[111] C. Ringeval and T. Suyama, J. Cosmol. Astropart. Phys. 12 (2017) 027.

[112] J. J. Blanco-Pillado, K. D. Olum, and X. Siemens, Phys. Lett. B 778, 392 (2018).

[113] B. P. Abbott et al. (LIGO Scientific and Virgo Collaborations), Phys. Rev. D 97, 102002 (2018).

[114] T. W. B. Kibble, J. Phys. A 9, 1387 (1976).

[115] N. Turok and D. N. Spergel, Phys. Rev. Lett. 66, 3093 (1991).

[116] R. Durrer, M. Kunz, and A. Melchiorri, Phys. Rep. 364, 1 (2002).

[117] D. G. Figueroa, M. Hindmarsh, and J. Urrestilla, Phys. Rev. Lett. 110, 101302 (2013).

[118] L. M. Krauss, Phys. Lett. B 284, 229 (1992).

[119] K. Jones-Smith, L. M. Krauss, and H. Mathur, Phys. Rev. Lett. 100, 131302 (2008).

[120] E. Fenu, D. G. Figueroa, R. Durrer, and J. Garcia-Bellido, J. Cosmol. Astropart. Phys. 10 (2009) 005.

[121] J. T. Giblin, Jr., L. R. Price, X. Siemens, and B. Vlcek, J. Cosmol. Astropart. Phys. 11 (2012) 006.

[122] D. G. Figueroa, Aspects of reheating, Ph.D. thesis, Universidad Autónoma de Madrid, 2010.

[123] M. Hindmarsh, J. Lizarraga, A. Lopez-Eiguren, and J. Urrestilla, Phys. Rev. Lett. 124, 021301 (2020).

[124] K. Dasgupta, J. P. Hsu, R. Kallosh, A. D. Linde, and M. Zagermann, J. High Energy Phys. 08 (2004) 030.

[125] C. P. Burgess, J. P. Conlon, L.-Y. Hung, C. H. Kom, A. Maharana, and F. Quevedo, J. High Energy Phys. 07 (2008) 073.

[126] D. J. E. Marsh, Phys. Rep. 643, 1 (2016).

[127] M. Maggiore, Gravitational Waves. Vol. 1, Oxford Master Series in Physics (Oxford University Press, New York, 2007).

[128] N. Bevis, M. Hindmarsh, M. Kunz, and J. Urrestilla, Phys. Rev. D 75, 065015 (2007).

[129] J. Lizarraga, I. Sendra, and J. Urrestilla, Phys. Rev. D 86, 123014 (2012).

[130] D. Daverio, M. Hindmarsh, M. Kunz, J. Lizarraga, and J. Urrestilla, Phys. Rev. D 93, 085014 (2016); 95, 049903(E) (2017).

[131] M. Hindmarsh, K. Rummukainen, and D. J. Weir, Phys. Rev. Lett. 117, 251601 (2016).

[132] M. Hindmarsh, J. Lizarraga, J. Urrestilla, D. Daverio, and M. Kunz, Phys. Rev. D 99, 083522 (2019).

[133] N. Bevis, M. Hindmarsh, M. Kunz, and J. Urrestilla, Phys. Rev. D 82, 065004 (2010).

[134] R. Durrer and M. Kunz, Phys. Rev. D 57, R3199 (1998). 
[135] D. Boyanovsky and H. J. de Vega, Phys. Rev. D 61, 105014 (2000).

[136] N. Bevis, M. Hindmarsh, and M. Kunz, Phys. Rev. D 70, 043508 (2004).

[137] J. Urrestilla, N. Bevis, M. Hindmarsh, M. Kunz, and A. R. Liddle, J. Cosmol. Astropart. Phys. 07 (2008) 010.

[138] W. H. Press, B. S. Ryden, and D. N. Spergel, Astrophys. J. 347, 590 (1989).

[139] A. Lopez-Eiguren, J. Urrestilla, and A. Achúcarro, J. Cosmol. Astropart. Phys. 01 (2017) 020; 06 (2017) E01.

[140] D. N. Spergel, N. Turok, W. H. Press, and B. S. Ryden, Phys. Rev. D 43, 1038 (1991).

[141] R. Durrer, M. Kunz, and A. Melchiorri, Phys. Rev. D 59, 123005 (1999).

[142] D. Bennett and S. Rhie, Phys. Rev. Lett. 65, 1709 (1990).

[143] A. Achucarro and J. Urrestilla, Phys. Rev. Lett. 85, 3091 (2000).

[144] Y. Akrami et al. (Planck Collaboration), Astron. Astrophys. 641, A10 (2020).

[145] P. A. R. Ade et al. (BICEP2 and Keck Array Collaborations), Phys. Rev. Lett. 121, 221301 (2018).

[146] V. Corbin and N. J. Cornish, Classical Quantum Gravity 23, 2435 (2006).

[147] K. Yagi and N. Seto, Phys. Rev. D 83, 044011 (2011); 95, 109901(E) (2017).

[148] S. Kawamura et al., Classical Quantum Gravity 28, 094011 (2011).

[149] S. Sato et al., J. Phys. Conf. Ser. 840, 012010 (2017).

[150] A. Vaquero, J. Redondo, and J. Stadler, J. Cosmol. Astropart. Phys. 04 (2019) 012.

[151] V. B. Klaer and G. D. Moore, J. Cosmol. Astropart. Phys. 10 (2017) 043.

[152] K. Saikawa, Universe 3, 40 (2017).
[153] F. Ferrer, E. Masso, G. Panico, O. Pujolas, and F. Rompineve, Phys. Rev. Lett. 122, 101301 (2019).

[154] M. Gorghetto, E. Hardy, and G. Villadoro, J. High Energy Phys. 07 (2018) 151.

[155] A. J. Long and L.-T. Wang, Phys. Rev. D 99, 063529 (2019).

[156] M. Buschmann, J. W. Foster, and B. R. Safdi, Phys. Rev. Lett. 124, 161103 (2020).

[157] S. Kuroyanagi, T. Hiramatsu, and J. Yokoyama, J. Cosmol. Astropart. Phys. 02 (2016) 023.

[158] C.-F. Chang and Y. Cui, Phys. Dark Universe 29, 100604 (2020).

[159] R. A. Battye and E. P. S. Shellard, Nucl. Phys. B423, 260 (1994).

[160] C. J. A. P. Martins and E. P. S. Shellard, Phys. Rev. D 54, 2535 (1996).

[161] C. J. A. P. Martins and E. P. S. Shellard, Phys. Rev. D 65, 043514 (2002).

[162] C. J. A. P. Martins, Phys. Lett. B 788, 147 (2019).

[163] R. A. Battye and E.P.S. Shellard, Recent perspectives on axion cosmology in Proceedings, International Heidelberg Conference on Dark Matter in Astro and Particle Physics (DARK 1996): Heidelberg, Germany (1996), pp. 554-579.

[164] M. Hindmarsh, J. Lizarraga, J. Urrestilla, D. Daverio, and M. Kunz, Phys. Rev. D 96, 023525 (2017).

[165] A. Saurabh, T. Vachaspati, and L. Pogosian, Phys. Rev. D 101, 083522 (2020).

[166] A. H. Jaffe, Phys. Rev. D 49, 3893 (1994).

[167] M. Kunz and R. Durrer, Int. J. Theor. Phys. 36, 2489 (1997).

[168] D. G. Figueroa, J. Garcia-Bellido, and A. Rajantie, J. Cosmol. Astropart. Phys. 11 (2011) 015. 